\title{
Selectivity via Cooperativity: Preferential Stabilization of the p65/ 14-3-3 Interaction with Semisynthetic Natural Products
}

\author{
Madita Wolter, Pim de Vink, João Filipe Neves, Sonja Srdanović, Yusuke Higuchi, Nobuo Kato, \\ Andrew Wilson, Isabelle Landrieu, Luc Brunsveld, and Christian Ottmann*
}

Cite This: J. Am. Chem. Soc. 2020, 142, 11772-11783

Read Online

\section{ACCESS | Llll Metrics \& More | 回 Article Recommendations | (s) Supporting Information}

ABSTRACT: Natural compounds are an important class of potent drug molecules including some retrospectively found to act as stabilizers of protein-protein interactions (PPIs). However, the design of synthetic PPI stabilizers remains an understudied approach. To date, there are limited examples where cooperativity has been utilized to guide the optimization of a PPI stabilizer. The 14-3-3 scaffold proteins provide an excellent platform to explore PPI stabilization because these proteins mediate several hundred PPIs, and a class of natural compounds, the fusicoccanes, are known to stabilize a subset of 14-3-3 protein interactions. 14-3-3 has been reported to negatively regulate the p65 subunit of the $\mathrm{NF}-\kappa \mathrm{B}$ transcription factor, which qualifies this protein complex as

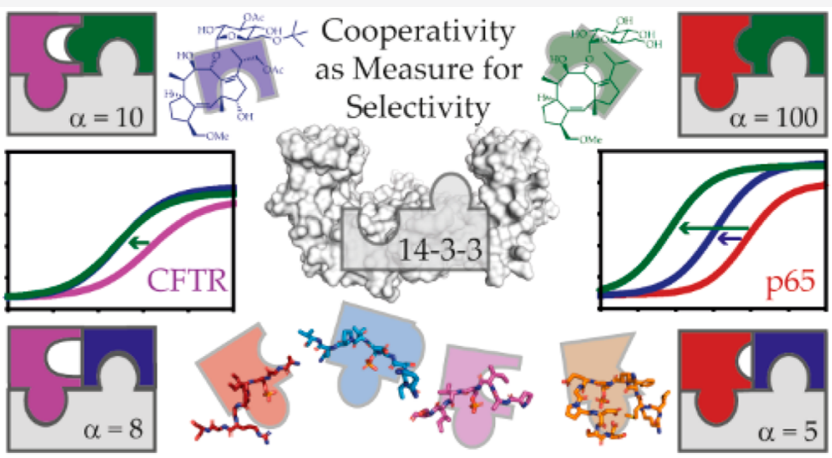
a potential target for drug discovery to control cell proliferation.

Here, we report the high-resolution crystal structures of two 14-3-3 binding motifs of p65 in complex with 14-3-3. A semisynthetic natural product derivative, DP-005, binds to an interface pocket of the p65/14-3-3 complex and concomitantly stabilizes it. Cooperativity analyses of this interaction, and other disease relevant 14-3-3-PPIs, demonstrated selectivity of DP-005 for the p65/ 14-3-3 complex. The adaptation of a cooperative binding model provided a general approach to characterize stabilization and to assay for selectivity of PPI stabilizers.

\section{INTRODUCTION}

The need to develop new strategies that address the challenges of today's most important diseases has motivated efforts to modulate protein-protein interactions (PPIs). ${ }^{1-4}$ PPI stabilizers are increasingly attractive given the potential for extending the druggable proteome, exploiting the endogenous-ligand uncompetitive mode of binding, and harnessing cooperativity as the driving force to enhance physiological, regulatory mechanisms instead of their artificial disruption. ${ }^{1-4}$ Promising progress has been made on both the inhibition and the stabilization of PPIs, although the ab initio design of PPI stabilizers in particular is still an exception. ${ }^{3}$ Nature itself provides the most potent and selective stabilizers of PPIs, which validate the concept of stabilization as a valuable strategy for targeting PPIs. ${ }^{5,6}$ The mode of action of known PPI stabilizers was mostly discovered retrospectively, as, for example, with rapamycin (Rapamune, Pfizer), cyclosporin (Sandimmun, Novartis Pharmaceuticals), or FK506 (Prograf, Astellas Pharma). ${ }^{5-7}$

To achieve PPI stabilization, the binding equilibrium of two target proteins needs to be shifted to the complexed state by binding of a third interaction partner, preferably a drug-like small molecule. This underlying binding improvement can be described via cooperative binding models whereby the increased binding affinity of the interaction partners, due to the formation of a ternary complex, is quantified via the cooperativity factor $\alpha .^{8-11}$ Adapting the cooperativity model to PPI drug discovery projects has been an underexplored opportunity, which has however started to gain more attention since PROTACs (proteolysis-targeting chimeras) were shown to induce cooperative binding of their protein binding partners. ${ }^{12-14}$ In this work, using the interactions of 14-3-3 proteins as an example, we show that cooperativity analysis not only provides a description of binding properties but can also be used as a direct entry to establishing the selectivity of compound-stabilized PPIs.

The 14-3-3 family of proteins pertains to the adaptor proteins, which represent one of the most important protein classes in the PPI field, because they mediate interactions between proteins and thereby regulate the function of their partner proteins. ${ }^{15,16}$ The 14-3-3 proteins recognize and bind

Received: February 23, 2020

Published: June 5, 2020 
phosphoserine/threonine motifs of hundreds of protein partners in eukaryotic cells, and subsequently alter the catalytic activity, subcellular localization, or interactional preference of their partners. ${ }^{17,18} 14-3-3$ is functionally present as a w-shaped dimer featuring two highly conserved, amphipathic grooves, where the phosphorylated residues are bound. ${ }^{19}$ A key element for the promising development of 14-3-3 proteins as potential drug targets is the availability of multiple members of a class of natural products, the fusicoccanes (FCs), that have been demonstrated to stabilize the binding of 14-3-3 proteins to a number of partner proteins. ${ }^{20-24}$ Protein crystallography studies have shown, for example, how such FCs stabilize 143-3 binding to protein targets such as $\mathrm{p} 53,{ }^{20} \mathrm{c}-\mathrm{Raf},{ }^{21} \mathrm{ER} \alpha{ }^{22}$ $\mathrm{Gab}_{2}{ }^{23}$ and CFTR. ${ }^{24}$ These natural compounds bind to protein pockets delineated by the interface of the complex of 14-3-3 and its partner protein. In this way, FCs establish contacts to both protein partners simultaneously and act as a molecular glue. ${ }^{5,25}$

Among the 14-3-3 partners are at least two proteins that crucially participate in the NF- $\kappa$ B pathway. ${ }^{26-28}$ The NF- $\kappa$ B signaling pathway has raised considerable attention as a therapeutic target, because of its intimate involvement in cell proliferation, apoptosis, immunity, and inflammation via the expression of several hundred genes. ${ }^{29-31} \mathrm{NF}-\kappa \mathrm{B}$ proteins are dimeric transcription factors, which are sequestered out of the nucleus into the cytosol by inhibitor proteins. Upon activation of the NF- $\kappa \mathrm{B}$ pathway, for example, by $\mathrm{TNF} \alpha, \mathrm{NF}-\kappa \mathrm{B}$ translocates to the nucleus and activates transcription (Figure 1A). Inhibition of this transcriptional activity has been the goal of numerous drug discovery campaigns; however, only a limited number of inhibitors of the NF- $\kappa \mathrm{B}$ pathway are currently marketed drugs, none of which directly target NF$\kappa \mathrm{B} .^{32-34}$ The NF- $\kappa \mathrm{B}$ subunit $\mathrm{p} 65$ and its inhibitor $\mathrm{I} \kappa \mathrm{B} \alpha$ bind to 14-3-3, which favors localization of p65 in the cytosol. ${ }^{26}$ Only limited information about the p65/14-3-3 interaction is available, even though downregulation of 14-3-3 leads to increased transcriptional activity of p65 in both a breast cancer model $^{27}$ and studies on Ischemia-Reperfusion. ${ }^{28}$ Stabilization of the p65/14-3-3 complex might therefore inhibit the transcriptional activity of p65, opening a new and unique opportunity for p65-specific NF- $\kappa \mathrm{B}$ inhibition.

In this study, we provide the structural and biophysical data and analysis to substantiate the biological interaction studies of the p65/14-3-3 interaction. We tested several natural and semisynthetic FCs as potential small-molecule stabilizers of this intriguing PPI, which revealed the semisynthetic DP-005 as the most active compound. A detailed analysis of the cooperative binding of the FCs, and DP-005 in particular, to the p65/14-3-3 interaction and multiple other disease relevant 14-3-3 PPIs was performed. DP-005 was at least 10-fold more active in stabilizing the p65/14-3-3 interaction than any other tested combination of FC and 14-3-3 PPI. The analysis clearly demonstrates that the cooperativity factor $\alpha$ can be used to quantify these stabilizing effects and is an underlying factor in achieving selectivity of stabilizer molecules on PPIs.

\section{RESULTS AND DISCUSSION}

Interaction of p65 and 14-3-3. In p65, the sequence surrounding three serines, S45, S281, and S340, matches to 143-3 consensus binding motifs (Figure 1B). ${ }^{19}$ Mutations of these serine residues to alanines were previously shown to reduce p65 binding to 14-3-3 in cells and to increase the p65 concentration in the nucleus. ${ }^{26}$ The S45 and S281 sites are
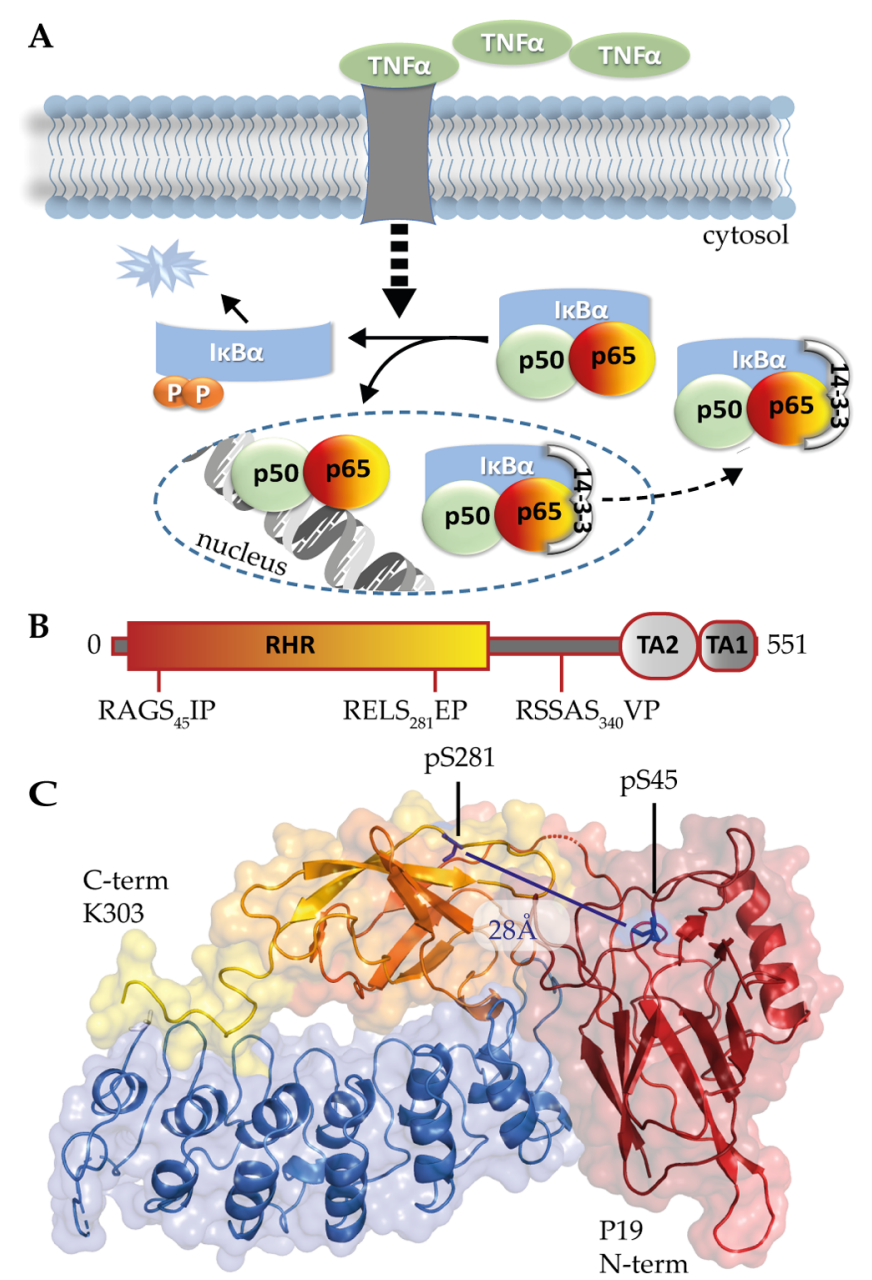

Figure 1. Interaction of $\mathrm{p} 65$ and 14-3-3. (A) Schematic representation of the interaction of 14-3-3, p65, and $\mathrm{I} \kappa \mathrm{B} \alpha$ in the context of the NF- $\kappa \mathrm{B}$ pathway. Briefly, upon activation of the pathway (for example, by TNF $\alpha$ ), a signal cascade (represented by a thick dashed arrow) leads to the phosphorylation and degradation of $\mathrm{I} \kappa \mathrm{B} \alpha$ and the nuclear translocation of NF- $\kappa \mathrm{B}$ (here represented by $\mathrm{p} 50 /$ $\mathrm{p} 65)$. Binding of $\mathrm{I} \kappa \mathrm{B} \alpha$ and 14-3-3 to p65 is necessary for nuclear export or cytosolic retention (narrow dashed arrow). (B) Domain representation of the p65 protein, with the Rel Homology Region (RHR), the two transactivator domains (TA1, TA2), and amino acid sequences of the three conserved potential 14-3-3 binding sites. (C) Cartoon representation of the crystal structure of the complex of $\mathrm{I} \kappa \mathrm{B} \alpha$ with the RHR domain of $\mathrm{p} 65$, with van der Waals' transparent surface. p65 (red to yellow) with I $\kappa \mathrm{B} \alpha$ (blue) (PDB ID: $1 \mathrm{IKN}$; p50 hidden for clarity; S45 and S281 are highlighted for clarity).

phosphorylated in response to TNF $\alpha$ treatment, ${ }^{35}$ and both are highly conserved within mammals. No TNF $\alpha$-dependent phosphorylation has been reported for the $\mathrm{S} 340$ site, ${ }^{35}$ which is also less conserved (Figure S1). The pS45 and pS281 sites are located in unstructured loop regions of $\mathrm{p} 65$, and the folding of p65 brings these two residues into proximity with each other, which implies the possibility for a bivalent interaction with the 14-3-3 dimer (Figure 1C). ${ }^{24,36,37}$

To represent these interaction motifs, peptides of 13 amino acids were synthesized for the pS45 and pS281 sites (Table 1). Noteworthy, for the pS45 site, two different sequences are available: whereas the canonical sequence features a glutamate at position 49, a second sequence identified an arginine instead. ${ }^{38}$ Because no biological or phenotypic relevance of 
Table 1. Overview of Synthetic Peptides Representing the Proposed 14-3-3 Binding Motifs of p65

\begin{tabular}{|c|c|c|}
\hline name & phosphosite & peptide sequence \\
\hline p $65 \_45^{\mathrm{E}}$ & $\mathrm{pS} 45$ & $\mathrm{EGRSAGpS}_{45} \mathrm{IPGE}_{49} \mathrm{RS}$ \\
\hline p $65 \_45^{\mathrm{R}}$ & $\mathrm{p} S 45$ & EGRSAGpS $_{45} \mathrm{IPGR}_{49} \mathrm{RS}$ \\
\hline p65_281 & pS281 & PSDRELpS $_{281}$ EPMEFQ \\
\hline p65_bi ${ }^{\mathrm{E}}$ & pS45pS281 & EGRSAGpS $_{45} \mathrm{IPGE}_{49} \mathrm{RSGSGGGSGPSDRELpS}_{281}$ EPMEFQ \\
\hline p65_bi ${ }^{\mathrm{R}}$ & pS45pS281 & EGRSAGpS $_{45}$ IPGR $_{49}$ RSGSGGGSGPSDRELpS $_{281}$ EPMEFQ \\
\hline
\end{tabular}
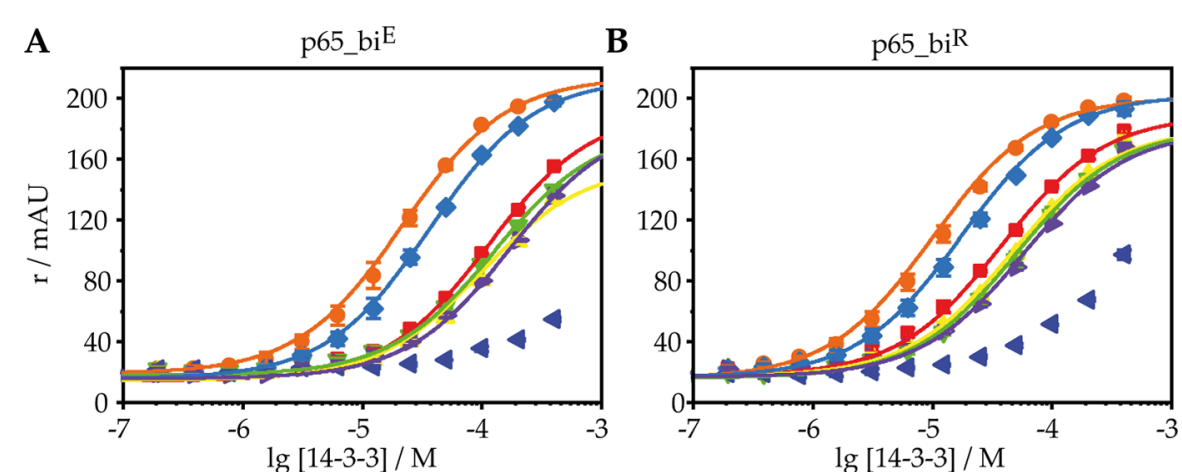

\begin{tabular}{l|cc} 
C & p65_bi & $\mathrm{K}_{\mathrm{D}}(\mu \mathrm{M})$ \\
& $\mathrm{E} 49$ & $\mathrm{R} 49$ \\
\hline$\beta$ & $112.6 \pm 14.9$ & $37.7 \pm 2.6$ \\
$\gamma$ & $21.6 \pm 1.8$ & $10.0 \pm 1.9$ \\
$\varepsilon$ & $98.6 \pm 34.1$ & $46.6 \pm 9.7$ \\
$\bar{\zeta}$ & $126.7 \pm 19.3$ & $50.7 \pm 10.2$ \\
$\eta$ & $36.7 \pm 3.8$ & $17.5 \pm 3.3$ \\
$\sigma$ & $>500$ & $>200$ \\
$\tau$ & $171.9 \pm 13.8$ & $58.0 \pm 11.5$
\end{tabular}
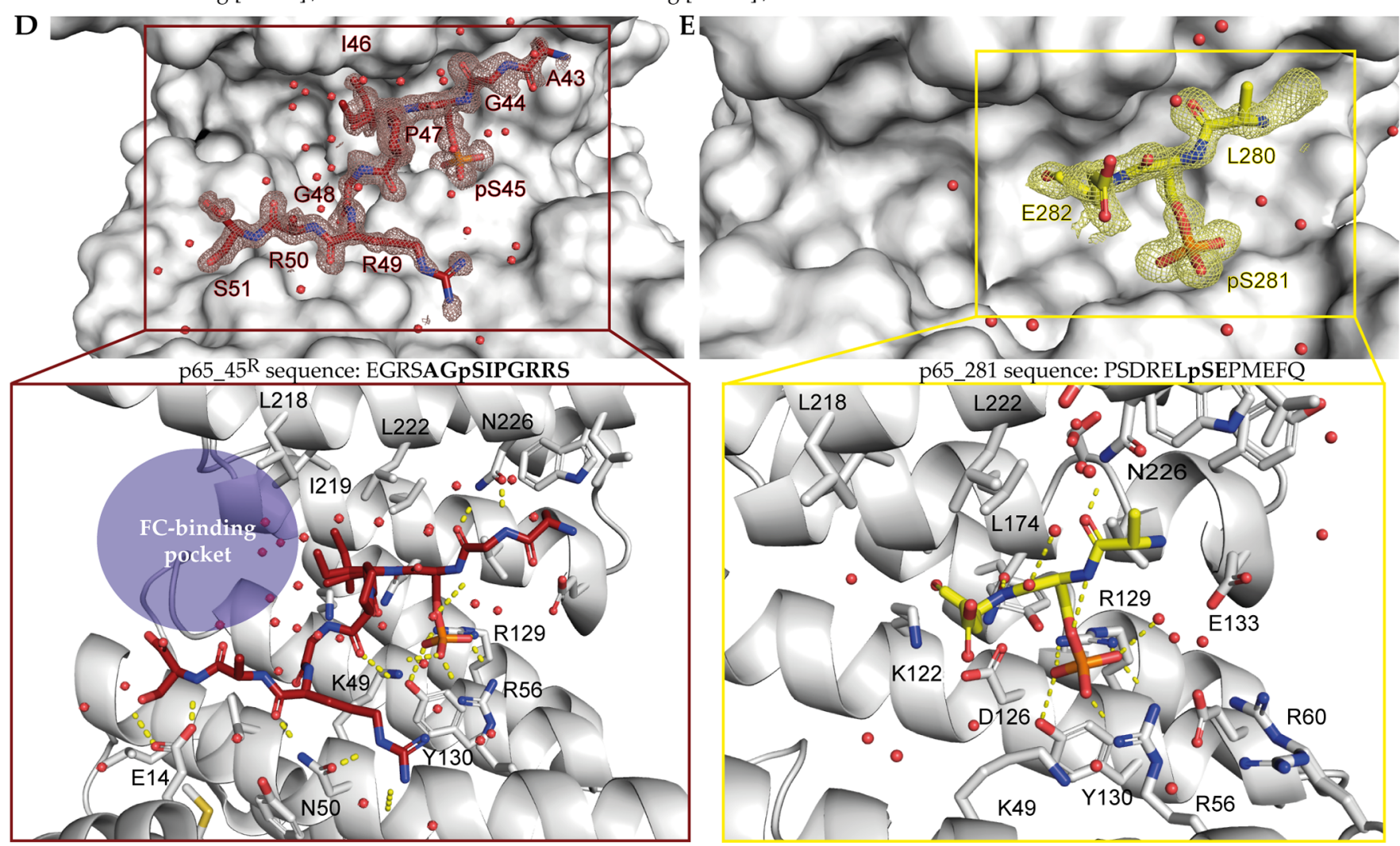

Figure 2. Binary complex of p65 peptides and 14-3-3. (A,B) The binding affinities of the indicated bivalent p65 peptides (100 nM) to all human 14-3-3 isoforms were measured via fluorescence anisotropy $\left(r\right.$ in mAU) assays. (C) $K_{\mathrm{D}}$ values in $\mu \mathrm{M}$ of the p65 bi ${ }^{\mathrm{E}}$ and p65 bi ${ }^{\mathrm{R}}$ to the different 14-3-3 isoforms as the mean $\pm \mathrm{SD}$. (D) High-resolution X-ray crystal structures of p65_45 (PDB ID: 6QHL). The peptide shows a C-terminal curved conformation, which leaves the typical FC binding pocket accessible (blue circle). (E) High-resolution X-ray crystal structures of p65 281 (PDB ID: 6QHM) in complex with 14-3-3 $\sigma \Delta \mathrm{C}$. The proteins are displayed as either a white surface or a cartoon (close up); water molecules are shown as red spheres. The $2 m F_{o}-F_{c}$ electron density map is displayed with $\sigma=1$. Polar contacts between protein and peptides are indicated as yellow dashed lines. Sequence of the p65_45 ${ }^{\mathrm{R}}$ peptide, EGRSAGpSIPGRRS; sequence of the p65_281 peptide, PSDRELpSEPMEFQ (residues visible in the electron density are bold).

these variants is reported, ${ }^{38}$ both sequences were tested for binding, hereafter termed $05545^{\mathrm{E}}$ and $\mathrm{p} 65 \_45^{\mathrm{R}}$ after the phosphorylation site $\mathrm{S} 45$ and the variant of amino acid 49. Each peptide is centered around the phosphorylated serine, flanked by six amino acids of the wild-type sequence on each side. To mimic the expected bivalent binding mode of the p65/14-3-3 complex, double phosphorylated peptides were synthesized, which connected the two binding sites with a flexible linker.

Elucidation of 14-3-3 Binding Motifs of p65. In a first step, the binding affinities of the two conserved 14-3-3 binding motifs of p65 were measured. To this end, the p65-peptides 


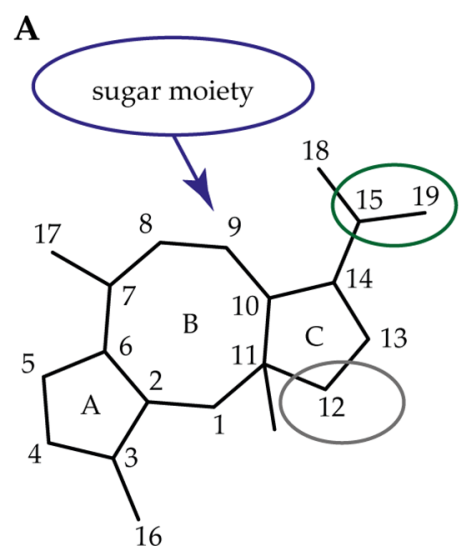

fusicoccane diterpene skeleton

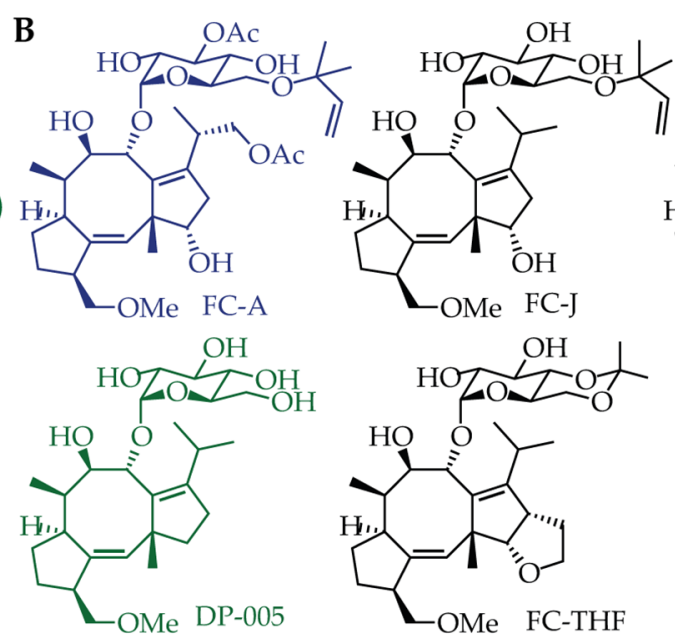

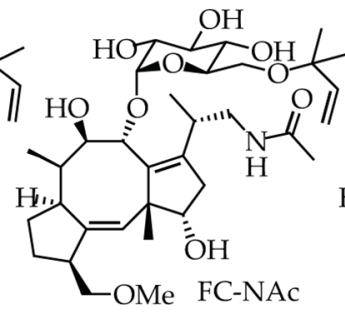

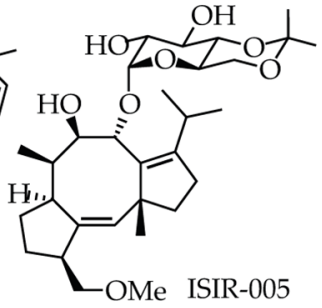

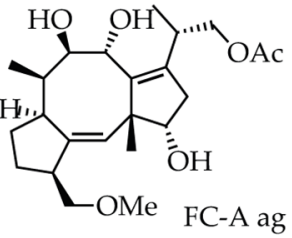

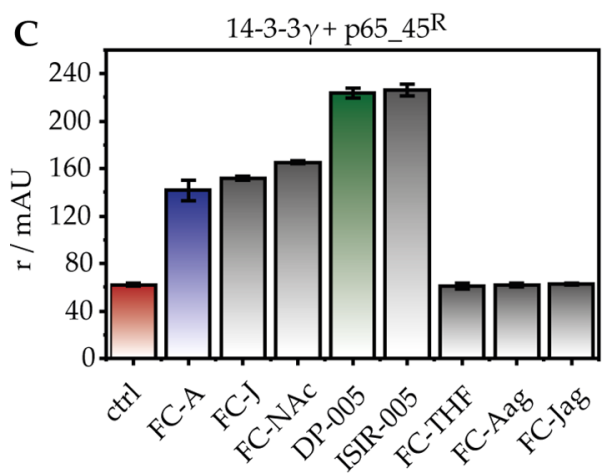

D

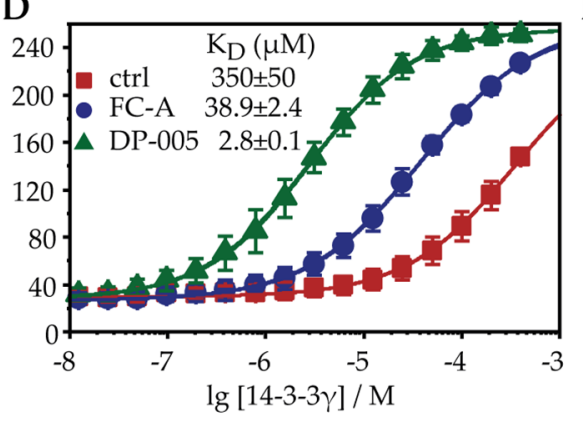

E

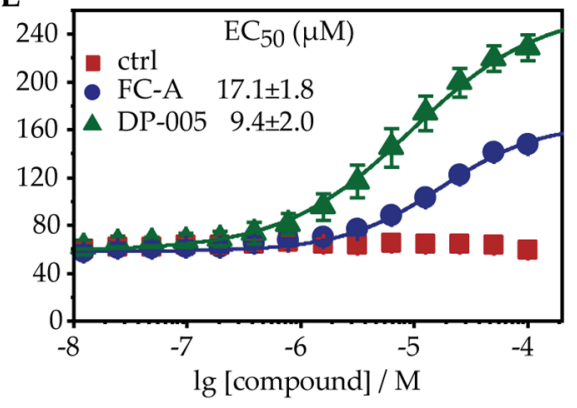

Figure 3. Stabilizing effect of fusicoccanes on the p65_45/14-3-3 interaction. (A) The diterpene skeleton of the fusicoccane family. Encircled are the positions that are of interest in this study. (B) A collection of eight FCs used to screen for a stabilizing effect on the p65/14-3-3 complex. (C) FA-based screening results of the FC-collection shown in (B) on the p65_45 $/ 14-3-3 \gamma$ complex. The anisotropy $(r$ in mAU) measurements were carried out with $100 \mu \mathrm{M}$ compound, $50 \mu \mathrm{M} 14-3-3 \gamma$, and $100 \mathrm{nM}$ of $\mathrm{p} 65 \_-45^{\mathrm{R}}$. Values represent the mean \pm SD of two independent measurements performed in technical triplicates. (D) Titration of $14-3-3 \gamma$ in the presence of $100 \mu \mathrm{M}$ compound or DMSO control (ctrl) and $100 \mathrm{nM}$ p65_45 measured with FA. Values and error bars represent the mean \pm SD of three independent singlet measurements. (E) Titration of compound or DMSO control (ctrl) in the presence of $50 \mu \mathrm{M} 14-3-3 \gamma$ and $100 \mathrm{nM} \mathrm{p65} \_45^{\mathrm{R}}$ measured with FA. Values and error bars represent the mean \pm SD of three independent singlet measurements.

were labeled with a fluorophore, and fluorescence anisotropy assays (FA) were performed. Both single site binding epitopes showed an increase in FA upon addition of 14-3-3 proteins, although not with full binding saturation (Figure S2). This confirms weak binding of both sites to all human 14-3-3 isoforms. The bivalent peptides featured a significantly stronger binding affinity to all 14-3-3 isoforms, with the p65_bi ${ }^{\mathrm{R}}$ binding roughly 2 -fold stronger than the $\mathrm{p} 65$ _bi ${ }^{\mathrm{E}}$ peptide (Figure 2A-C). The strongest binding was observed with 14-3-3 $\gamma\left(K_{\mathrm{E} 49}^{\mathrm{D}}=21.6 \pm 1.8 \mu \mathrm{M} ; K_{\mathrm{R} 49}^{\mathrm{D}}=10.0 \pm 1.9 \mu \mathrm{M}\right)$, directly followed by $14-3-3 \eta\left(K_{\mathrm{E} 49}^{\mathrm{D}}=36.7 \pm 3.8 \mu \mathrm{M} ; K_{\mathrm{R} 49}^{\mathrm{D}}=\right.$ $17.5 \pm 3.3 \mu \mathrm{M})$. The dissociation constants of $14-3-3 \beta, \varepsilon, \zeta$, and $\tau$ with p65_bi ${ }^{\mathrm{R}}$ ranged from 38 to $127 \mu \mathrm{M}$, while 14-3-3 $\sigma$ showed the weakest binding (Figure $2 \mathrm{C}$ ). The range of binding affinities for the 14-3-3 isoforms is not atypical and seems to be independent of the peptide sequence. ${ }^{24,39,40}$ The peptideprotein binding data thus confirm a physical interaction between p65 and 14-3-3 and argue in favor of a bivalent binding event.

The binding between p65 and 14-3-3 was further elucidated with structural data; cocrystallization of the single phosphorylated peptides with 14-3-3 $\sigma \Delta \mathrm{C}$ (last 17 unstructured residues on the C-terminus are truncated) resulted in crystals with the highest resolution of 1.2 and $1.25 \AA$ for the p $65 \_45^{\mathrm{R}}$ and p65_281 complexes, respectively (Table S1). The phosphorylated S45 makes polar contacts with R56, R129, and Y130, the general phospho-accepting pocket of 14-3-3 (Figure 2D; PDBID: 6QHL). Upstream of pS45 only A43 and G44 are visible in the electron density map, whereby the backbone of G44 makes polar contacts with N226 of the 14-3-3 protein. At the +1 position of $\mathrm{pS} 45$, the isoleucine points into a hydrophobic pocket of the 14-3-3 binding groove to make hydrophobic contacts with L174, I219, and L222. The presence of a proline residue at the +2 position results in a curved conformation in the binding groove, which potentially creates a ligandable interface pocket (Figure 2D). In comparable crystal structures of 14-3-3 with other interaction partners, a similar pocket accommodates FCs and their derivatives. ${ }^{20-24}$ The side chain of R49 makes two polar contacts with N50 of 14-3-3 as well as with several water molecules. The C-terminus of the $\mathrm{p} 65-45^{\mathrm{R}}$ peptide makes additional polar contacts with E14 of 14-3-3. The direct comparison of the two variants of the p65_45 peptide only shows small differences in binding affinity, and structural information could only be obtained for the p 65 _ $45^{\mathrm{R}}$ peptide; hence, this variant was selected for the next experiments.

The electron density of the p65_281 peptide in complex with 14-3-3 revealed only the backbone of two additional 


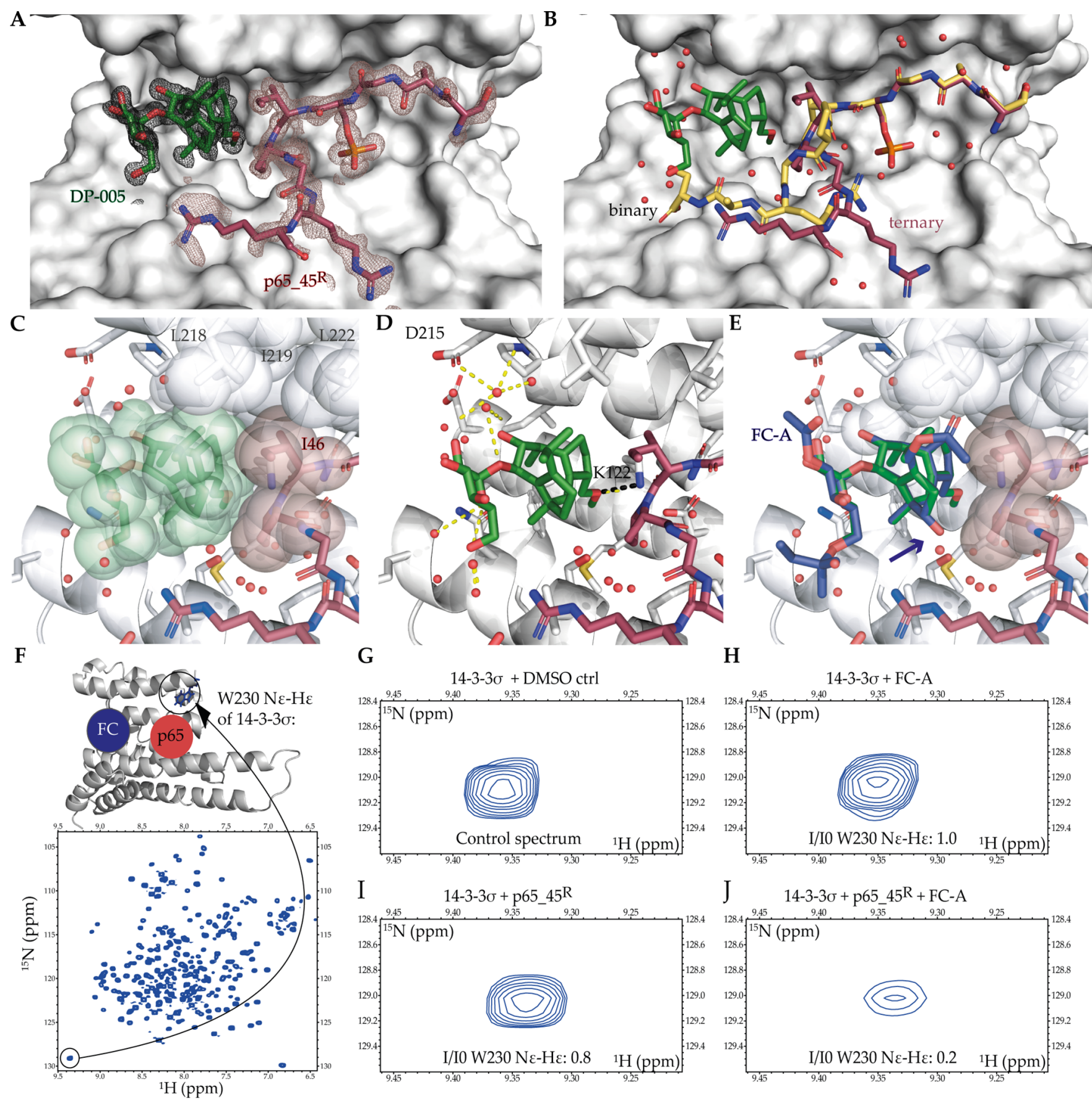

Figure 4. Structural analysis of the ternary DP-005/p65/14-3-3 and FC-A/p65/14-3-3 complexes. (A) Crystal structure of DP-005 (green sticks), p $6545^{\mathrm{R}}$ (red sticks), and 14-3-3 $\sigma \Delta \mathrm{C}$ (white surface) (PDB ID: 6NV2). (B) Binding of DP-005 induces a reorientation of the p65 $45^{\mathrm{R}}$ peptide (original conformation of binary complex, yellow sticks; ternary complex with DP-005, red sticks). (C) Hydrophobic contacts are indicated with spheres. (D) Polar contacts of DP-005 displayed as yellow dashed lines with the polar contact of the methoxy group of DP-005 and K122 highlighted with black dashed lines. (E) Overlay of FC-A (blue sticks) and DP-005 (green sticks). An arrow indicates the hydroxyl group at position 12, which causes the difference in affinity between both compounds. Hydrophobic residues of the peptide/protein complex are shown as spheres. (F) The resonance corresponding to the W230 $\mathrm{N} \varepsilon-\mathrm{H} \varepsilon$ bond of 14-3-3 $\sigma$ was monitored to detect the stabilization of this PPI. This resonance is circled in black on the ${ }^{1} \mathrm{H}-{ }^{15} \mathrm{~N}$ TROSY-HSQC spectrum of ${ }^{15} \mathrm{~N}^{13} \mathrm{C}^{2} \mathrm{H}$-labeled 14-3-3 $\sigma$ (shown on the right), and the corresponding residue W230 is represented as sticks, colored in blue and circled in black on the crystal structure (shown on the left, represented as a white surface). This residue is close to the peptide-binding site and distant from the FC-A binding site. (G-J) The enlarged spectral region of the ${ }^{1} \mathrm{H}-{ }^{15} \mathrm{~N}$

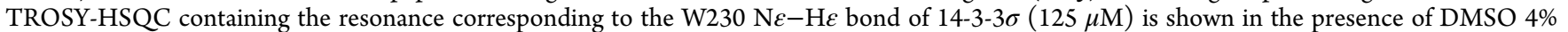
$(\mathrm{v} / \mathrm{v})$, present in all samples $(\mathrm{G}), \mathrm{FC}-\mathrm{A} 125 \mu \mathrm{M}(\mathrm{H}), \mathrm{p} 65 \_45^{\mathrm{R}}$ peptide $625 \mu \mathrm{M}(\mathrm{I})$, and p65_45 $5^{\mathrm{R}}$ peptide $625 \mu \mathrm{M}$ and FC-A $125 \mu \mathrm{M}(\mathrm{J})$.

amino acids besides that of the phosphorylated serine (Figure 2E; PDB ID: 6QHM). Additional electron density on the Nterminus of the peptide could not be fully interpreted, which indicated multiple conformations of these amino acids. The phosphoserine is trapped in the binding groove via polar contacts with R59, R129, and Y130 of 14-3-3 $\sigma \Delta \mathrm{C}$. Additionally, contacts could also be observed with the backbone nitrogen of E282, at the +1 position of p65_pS281, and N175 
of 14-3-3 and the carbonyl of L280 of the peptide and N226 of the protein.

Fusicoccanes Stabilize the p65/14-3-3 Interaction. The structures of $\mathrm{p} 65 \_45^{\mathrm{R}}$ and $\mathrm{p} 65$ _281 provide the structural basis to investigate possible molecular strategies to stabilize these binary complexes. Because the 14-3-3 binding groove is highly conserved, the p65-peptide/14-3-3 $\sigma \Delta \mathrm{C}$ interface displays the key molecular details of the primary p65/14-3-3 interface. ${ }^{19}$ The $05545^{\mathrm{R}}$ peptide is bending in a way that the typical FC binding pocket remains accessible and the conformation of the p65_281 is unknown (Figure 2D,E). This leaves the possibility that both complexes may be stabilized by FCs or synthetic derivatives thereof. The FCs share a diterpene core with a $5(\mathrm{~A})-8(\mathrm{~B})-5(\mathrm{C})$ ring structure (Figure $3 \mathrm{~A}) ;^{41}$ modifications that include additional sugar moieties, hydroxylation, or acetylation have introduced considerable chemical diversity into this compound family (Figure 3B)..$^{42}$

The effect of FCs on the p65/14-3-3 complex was investigated with a small collection of eight natural FCs and their semisynthetic derivatives (Figure 3B). The tested FCs have a FC-A-like structure with a hydroxylation at position 8 , a methoxy group at position 16, and a sugar moiety at position 9 . The main variations within the collection used here are on the sugar moiety, position 12 and/or 19. FC-A contains an additional acetyl on the 19 position, while the natural product FC-J only has an isopropyl on the C-ring. A recent study showed that replacing the acetyl with an acetamide (FC-NAc) improves the affinity to $14-3-3$ with various partners. ${ }^{25}$ The 12 position is hydroxylated in the natural compounds, but this position is not hydroxylated in the semisynthetic derivatives DP-005 and ISIR-005, ${ }^{23,43}$ and a bulky group was introduced for FC-THF. ${ }^{44}$ For FC-A-aglycon (FC-A-ag) ${ }^{44}$ and FC-Jaglycon (FC-J-ag), ${ }^{44}$ the sugar moiety was removed. Initially, a single dose of compound and the protein concentration were tested using FA. The single phosphorylated peptides were used so that a potential stabilizing effect could be correlated to a specific binding motif. 14-3-3 $\gamma$ was used because of the highest binding affinity of all peptides to this isoform.

The FA results showed that several FCs have a stabilizing effect on the p65 $45^{\mathrm{E}} / 14-3-3 \gamma$ and $\mathrm{p} 65-45^{\mathrm{R}} / 14-3-3 \gamma$ interaction, while they do not stabilize the p65 281/14-3-3 $\gamma$ complex (Figure 3C and Figure S3). The fusicoccanes FC-A, FC-J, and FC-NAc elicited about a 2-fold higher anisotropy signal of the p65_45 $/ 14-3-3 \gamma$ PPI as compared to the DMSO control. Modeling of cooperative complex stabilization of FC/ 14-3-3 PPIs indicated an intrinsic affinity of 300-500 $\mu \mathrm{M}$ for FC-A and FC-J and about $10 \mu \mathrm{M}$ for FC-NAc. The higher affinity of FC-NAc to the apo14-3-3 does not translate into significantly higher stabilization of the p65 $45^{\mathrm{R}} / 14-3-3$ complex. ${ }^{11}$ These data show that the FC-induced stabilization is not directly connected to the intrinsic affinity of the FCs to 14-3-3. Interestingly, semisynthetic analogues DP-005 and ISIR-005 induced a stronger increase in the anisotropy signal (about 3-fold). Bulky extensions at the C-ring (FC-THF) and the absence of the sugar moiety (FC-A-ag and FC-J-ag) abrogated the ability to stabilize the p65_45 $/ 14-3-3 \gamma$ complex. A hydroxyl group at the 12-position or its ringextension thus causes a loss in activity. The negligible activity of the aglycons is in line with previous work on these synthetic variants, which generally show lower activity. ${ }^{44}$

The stabilizing effects of FC-A and DP-005 were further investigated via $14-3-3 \gamma$ protein titrations to the $\mathrm{p} 65 \_45^{\mathrm{R}}$ peptide at a set concentration of DP-005, FC-A, or the DMSO control (Figure 3D). The protein titrations confirmed the stronger stabilizing effect of DP-005 as compared to FC-A, with a clear left shift of the binding curve, to afford apparent $K_{\mathrm{D}}$ values of $2.8 \pm 0.1$ and $38.9 \pm 2.4 \mu \mathrm{M}$, respectively. Constraining the fitting for the DMSO control, while using the upper plateau of the DP-005 and FC-A binding curves, results in an apparent $K_{\mathrm{D}}$ of about $350 \mu \mathrm{M}$ for the p65_45 $/ 14-3-3 \gamma$ complex.

Hence, the improvement in peptide stabilization acquaints to approximately 10-fold (FC-A) and 100-fold (DP-005) as compared to the DMSO control. Finally, the effective concentrations of FC-A and DP-005 were determined by titrating the compounds to a fixed protein and peptide concentration of 50 and $0.1 \mu \mathrm{M}$, respectively. This gave an $\mathrm{EC}_{50}$ value of $9.4 \pm 2.0 \mu \mathrm{M}$ for the semisynthetic compound DP-005, while the natural compound FC-A showed a weaker activity with an $\mathrm{EC}_{50}$ value of $17.1 \pm 1.8 \mu \mathrm{M}$ (Figure $3 \mathrm{E}$ ).

Crystallography and NMR Provide Structural Confirmation of the FC Binding to p65/14-3-3. To corroborate the stabilization and SAR obtained using FA, we sought a structural analysis of the ternary complex of DP-005 with p65_45 $/ 14-3-3$. For this purpose, DP-005 was soaked into crystals of the preformed $\mathrm{p} 65$ 4 $45^{\mathrm{R}} / 14-3-3 \sigma \Delta \mathrm{C}$ complexes, to reveal extra electron density for the p65_45 $/ 14-3-3 \sigma \Delta \mathrm{C}$ crystals. This electron density allowed for the complete refinement of DP-005 (Figure 4A, Table S1, PDB ID: $6 \mathrm{NV} 2)$. The crystal structure provided detailed information about the orientation of the diterpenoid core and the sugar moiety. Of note, the orientation of the peptide changed significantly upon binding of DP-005 (Figure 4B). In particular, amino acid $\mathrm{P} 47$ changed from a trans- to a cisconformation, and the residues from G48 to S51 moved away from the ligand. The most prominent interactions between DP-005 and the protein complex are the hydrophobic contacts with $\mathrm{I} 46$ and P47 of p65_45 ${ }^{\mathrm{R}}$ as well as with L218, I219, and L222 of 14-3-3 $\sigma \Delta \mathrm{C}$ (Figure 4C). Noteworthy is the contact of the methoxy group of DP-005 with the $\varepsilon$-amino group of K122 of the protein, while the sugar moiety was surrounded by the water shell of $14-3-3 \sigma \Delta \mathrm{C}$ (Figure 4D). The fact that FCaglycons showed only a negligible activity might be due to the importance of this sugar-water shell interaction.

An overlay of FC-A with the conformation of DP-005 points out the impact of the singular hydroxyl group at the 12position of FC-A, not present in DP-005, on the binding to the protein complex (Figure 4E). The hydroxyl group would have an orientation that would induce a steric and polar clash with hydrophobic elements of the peptide. This indicates that the peptide would potentially require further rearrangements to accommodate the binding of FC-A, which potentially explains why the ternary structure of FC-A/p65_45 $5^{\mathrm{R}} / 14-3-3 \sigma \Delta \mathrm{C}$ could not be determined with X-ray crystallography.

As an alternative to obtaining structural information on the binding of FC-A to the binary p65/14-3-3 complex, NMR measurements were performed, on the basis of the assignments of $14-3-3 \sigma$ signals. ${ }^{45}$ Chemical shift perturbations in the resonances corresponding to specific residues along 14-3-3 $\sigma$ confirmed that FC-A binds in its previously reported binding pocket, in the presence and in the absence of the p65_45 peptide (Figure S4). To specifically monitor the binding event of the p65 $45^{\mathrm{R}}$ to $14-3-3 \sigma$, we focused on the ${ }^{1} \mathrm{H}-{ }^{15} \mathrm{~N}$ TROSY-HSQC signature of the residue W230 side chain of ${ }^{15} \mathrm{~N}^{13} \mathrm{C}^{2} \mathrm{H}$ labeled $14-3-3 \sigma$. This residue is specifically affected 


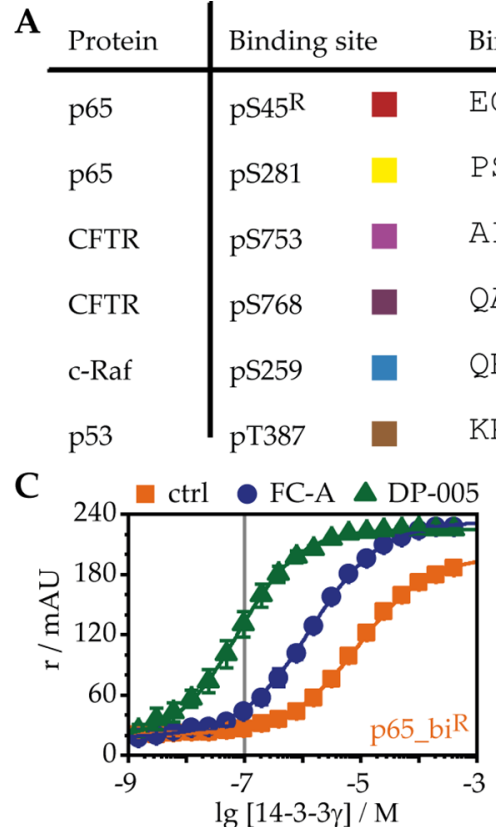

$\mathbf{D}$

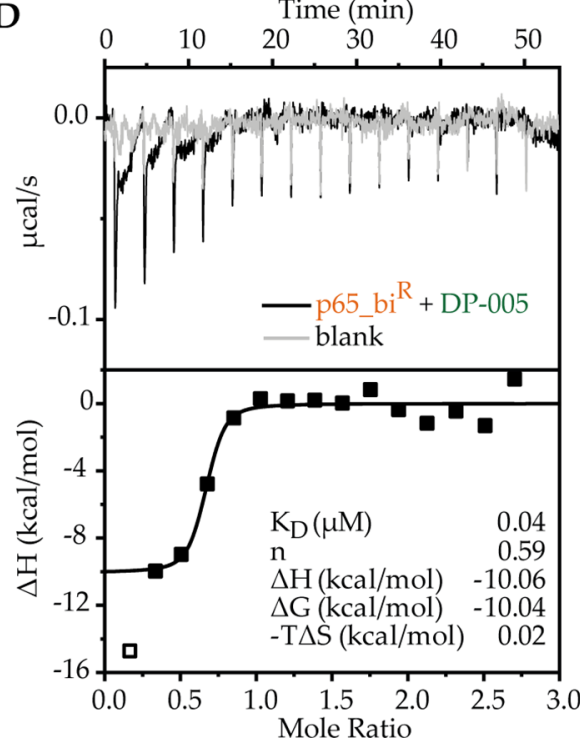

Binding Epitope $[-6 ; 6] \quad$ B

EGRSAG pS IPGRRS

PSDREL PS EPMEFQ

AILPRI pS VISTGP

QARRRQ PS VLNLMT

QRQRST PS TPNVHM

KKLMFK PT EGPDSD

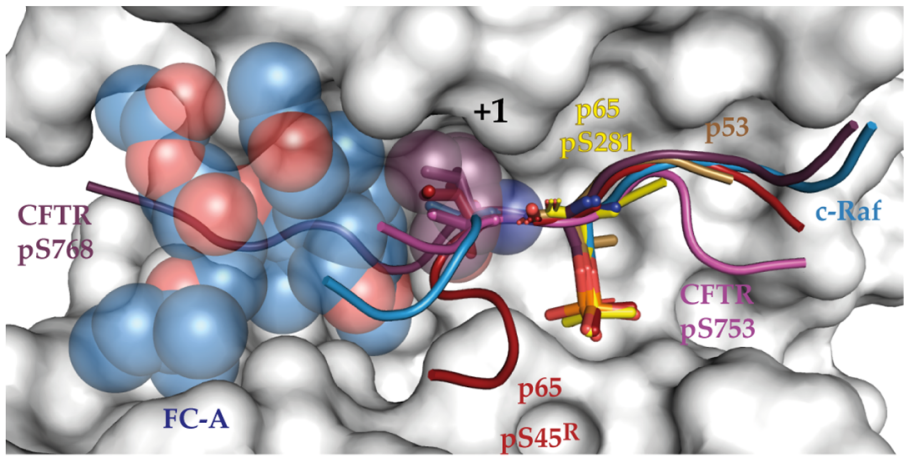

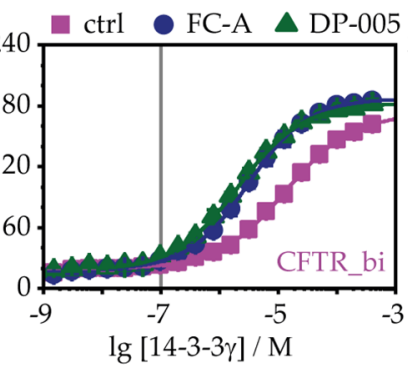

E
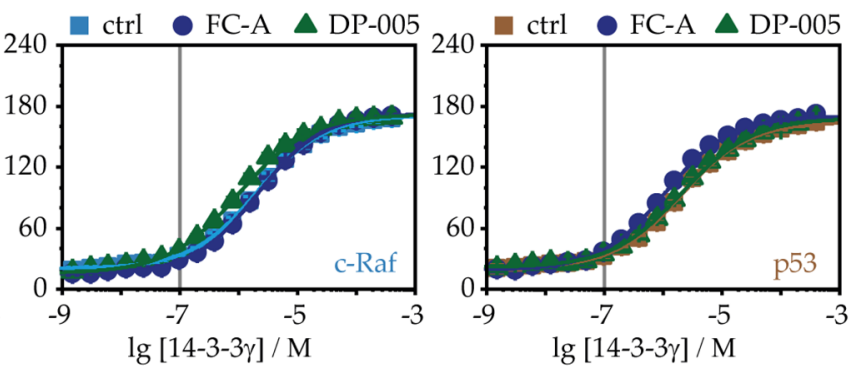

FA results

\begin{tabular}{l|rcc} 
KDapp $(\mu \mathrm{M})$ & \multicolumn{1}{|c}{ ctrl } & FC-A & DP-005 \\
\hline p65_45R & $350 \pm 50$ & $38.9 \pm 2.4$ & $2.8 \pm 0.1$ \\
p65_biR & $9.2 \pm 1.3$ & $1.4 \pm 0.1$ & $0.07 \pm 0.005$ \\
CFTR_bi & $13.3 \pm 0.5$ & $3.0 \pm 0.07$ & $2.1 \pm 0.05$ \\
c-Raf & $2.1 \pm 0.2$ & $2.1 \pm 0.07$ & $1.0 \pm 0.09$ \\
p53 & $2.0 \pm 0.1$ & $1.1 \pm 0.04$ & $2.1 \pm 0.1$
\end{tabular}

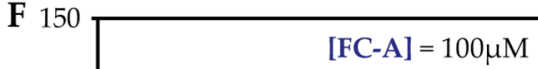

\begin{tabular}{l|ccc}
\multicolumn{4}{|c}{ ITC results } \\
$\mathrm{K}_{D^{\mathrm{app}}(\mu \mathrm{M})}$ & ctrl & FC-A & DP-005 \\
\hline p65_bi & $11.2 \pm 0.1$ & $2.3 \pm 1.2$ & $0.13 \pm 0.08$ \\
CFTR_bi & $11.9 \pm 2.1$ & n.d. & $2.33 \pm 0.73$ \\
c-Raf & $2.0 \pm 0.5$ & $1.9 \pm 0.10$ & $1.3 \pm 0.02$ \\
p53 & $1.2 \pm 0.2$ & $1.0 \pm 0.13$ & $1.3 \pm 0.4$
\end{tabular}

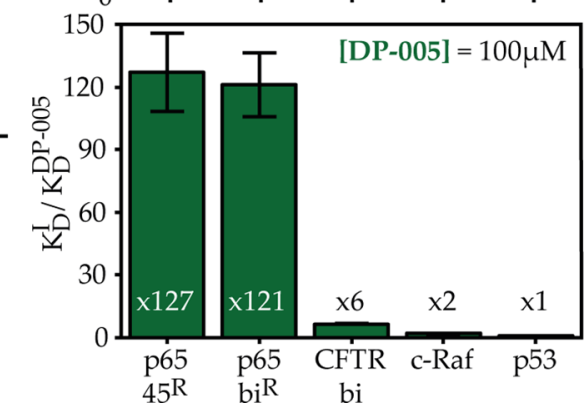

Figure 5. Comparison of FC-induced stabilization on 14-3-3 PPIs. (A) Overview of 14-3-3 binding epitopes. (B) Overlay of the crystal structures of the binding epitopes shown in (A) (cartoon representation, phosphorylated residue, and +1 amino acid shown as sticks). FC-A is shown as transparent spheres, while 14-3-3 is shown as white van der Waals surface. (C) Binding affinities of indicated peptides were measured for 14-3-3 $\gamma$ in the presence of DMSO as control (ctrl), $100 \mu \mathrm{M}$ FC-A, or $100 \mu \mathrm{M}$ DP-005 with FA ( $r$ in mAU). For peptide sequences, see Tables 1 and S3. (D) Apparent binding affinity of DP-005/p65_bi $\mathrm{i}^{\mathrm{R}} / 14-3-3 \gamma$ complex formation measured with ITC $(n=1)$. For the duplicate, see Figure S7C. Syringe, $150 \mu \mathrm{M}$ 14-3-3 $\gamma, 200 \mu \mathrm{M}$ DP-005; cell, $10 \mu \mathrm{M}$ p65_bi ${ }^{\mathrm{R}}, 200 \mu \mathrm{M}$ DP-005. (E) $K_{\mathrm{D}}{ }^{\text {app }}$ values in $\mu \mathrm{M}$ for the indicated peptides for the DMSO control (ctrl) or with FC-A or DP-005 as mean \pm SD measured with FA (upper panel, $n=3$ ) and ITC (lower panel, $n=2)$. n.d. $=$ not determined. (F) Increase in affinity of the binding partners due to FC-A or DP-005 shown as the ratio of the dissociation constant of the DMSO control $\left(K_{D}{ }^{I}\right)$ divided by the dissociation constant in the presence of either FC-A $\left(K_{\mathrm{D}}{ }^{\mathrm{FC}-\mathrm{A}}\right)$ or DP-005 $\left(K_{\mathrm{D}}{ }^{\mathrm{DP}-005}\right)$ based on FA results. The numbers indicate the factor with which the binding affinity is enhanced due to the FCs as compared to the control.

by the p $65 \_45^{\mathrm{R}}$ peptide binding, but not by the FC-A binding, as can be expected from its remote position from the FC-A binding pocket (Figure $4 \mathrm{~F}$ ). Its side-chain $\mathrm{N} \varepsilon-\mathrm{H} \varepsilon$ resonance has a clear signature because it is isolated in the spectrum (Figure 4F) and is unambiguously assigned (Figure S5). Monitoring the resonance intensity of $\mathrm{N} \varepsilon-\mathrm{H} \varepsilon$ W230 revealed that the intensity of this resonance is, as expected, not affected by the presence of FC-A alone (Figure 4G,H). Addition of the p $65 \_45^{\mathrm{R}}$ peptide alone results in resonance broadening due to the binding and an intensity drop to $80 \%$ as compared to the reference spectrum (Figure 4I). Addition of FC-A together with the p65_45 ${ }^{\mathrm{R}}$ peptide resulted in the most pronounced decrease of the intensity of the $\mathrm{N} \varepsilon-\mathrm{H} \varepsilon$ W230 resonance, down to $20 \%$ (Figure $4 \mathrm{~J}$ ). The data thus orthogonally confirm the stabilization of the p65_45 $/ 14-3-3 \sigma$ complex by FC-A.

Preferential Stabilization of the p65/14-3-3 Complex by DP-005. The stabilization of p65/14-3-3 by DP-005 was significantly stronger than the effect of FC-A (vide supra), which raised the question about DP-005's impact on other FC responsive 14-3-3 PPIs. Crucial differences between FC-A and 

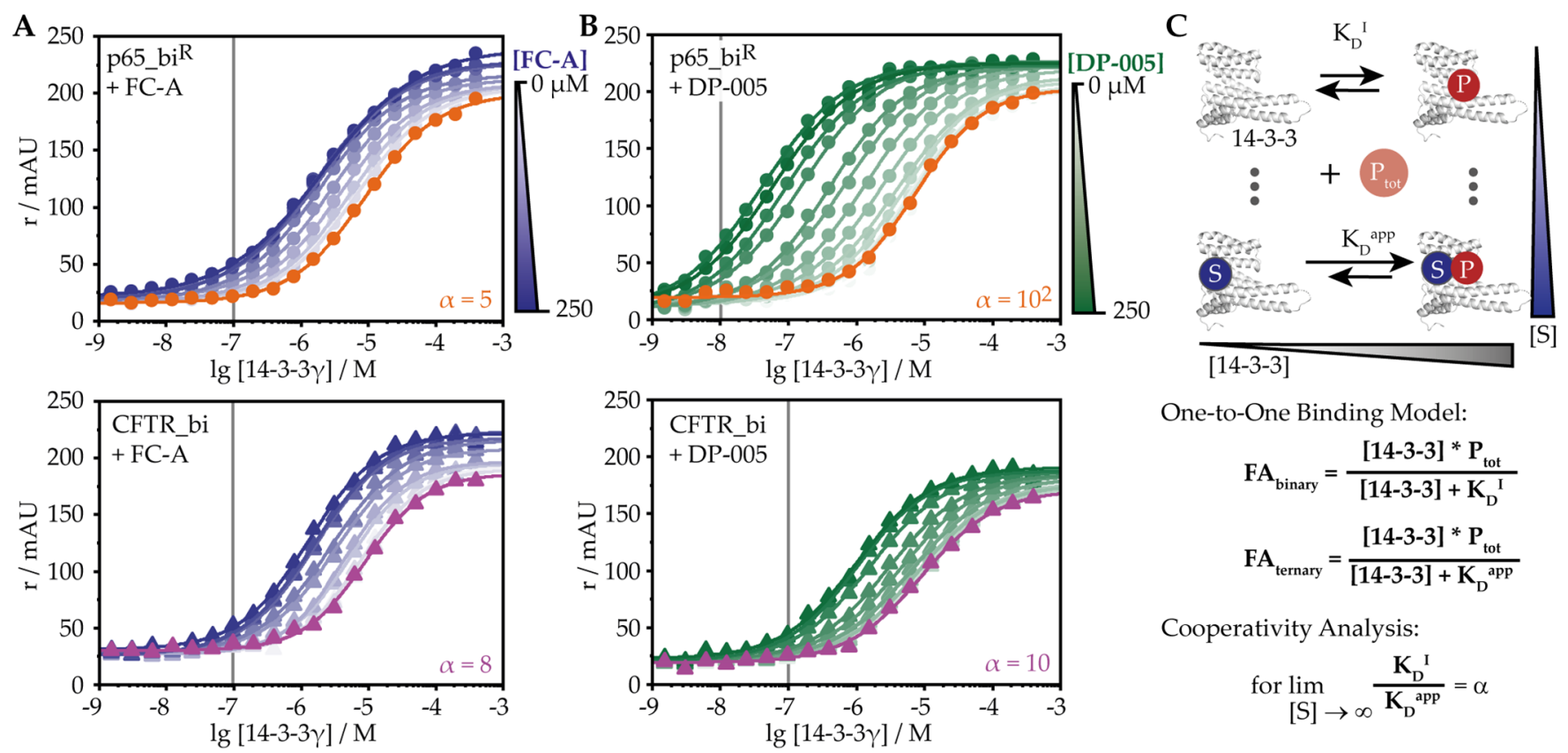

One-to-One Binding Model:

$$
\begin{aligned}
F A_{\text {binary }} & =\frac{[14-3-3] * P_{\text {tot }}}{[14-3-3]+K_{D}{ }^{\mathrm{I}}} \\
F A_{\text {ternary }} & =\frac{[14-3-3] * P_{\text {tot }}}{[14-3-3]+K_{D}^{\text {app }}}
\end{aligned}
$$

Cooperativity Analysis:

$$
\text { for } \lim _{[\mathrm{S}] \rightarrow \infty} \frac{\mathrm{K}_{\mathrm{D}}{ }^{\mathrm{I}}}{\mathrm{K}_{\mathrm{D}}^{\text {app }}}=\alpha
$$
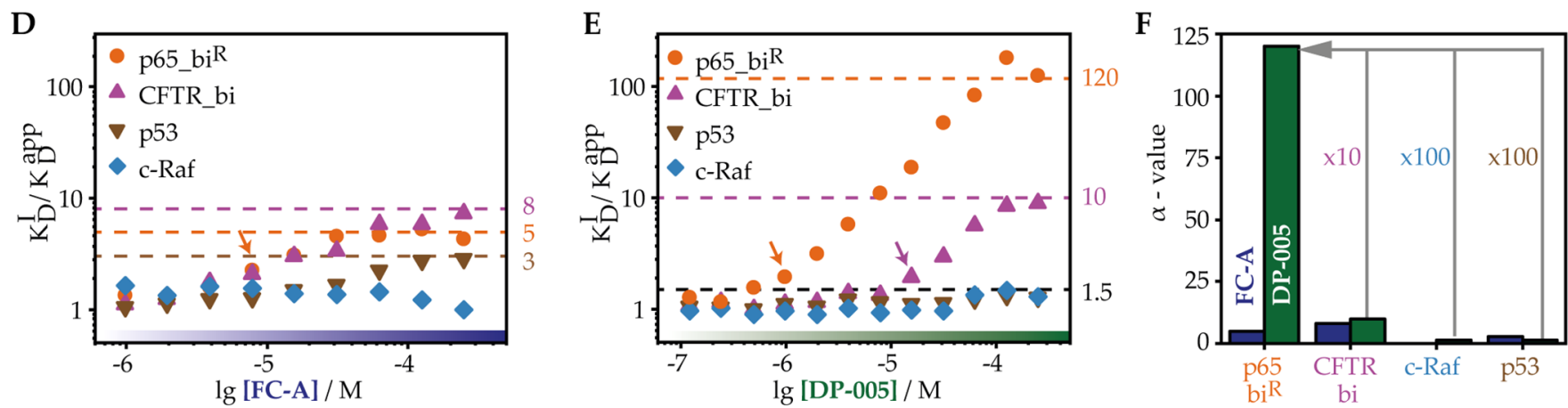

Figure 6. Cooperativity analysis of FC-A and DP-005 on 14-3-3 PPIs. (A) 2D FA-results of FC-A with p65_bi ${ }^{\mathrm{R}} / 14-3-3 \gamma$ and CFTR bi $(n=1)$. FC-A concentrations range from 0 to $250 \mu \mathrm{M}$; the vertical gray line indicates the peptide concentration. Anisotropy $(r)$ given in mAU. (B) 2D FAresults of DP-005 with p65_bi ${ }^{\mathrm{R}} / 14-3-3 \gamma$ or CFTR_bi $(n=1)$. DP-005 concentrations range from 0 to $250 \mu \mathrm{M}$; the vertical gray line indicates the peptide concentration. (C) Scheme of 2D titration in FA. 14-3-3 was titrated against $100 \mathrm{nM}$ of peptide (P) varying fixed concentrations of stabilizer (S). The data analysis was based on a one-to-one binding model. For cooperativity analysis, the ratio of $K_{\mathrm{D}}$ of the binary $\left(K_{\mathrm{D}}^{\mathrm{I}}\right)$ and ternary $\left(K_{\mathrm{D}}{ }^{\text {app}}\right)$ complex was used to derive the cooperativity factor $\alpha$ (for derivation of the equations and additional information, see the Supporting Information). (D) $K_{\mathrm{D}}^{\mathrm{I}} / K_{\mathrm{D}}{ }^{\text {app }}$ ratio plotted against FC-A concentration for $2 \mathrm{D}$ titrations shown in (A) and Figure S9. The arrow indicates the minimal active concentration of the stabilizer, while the curve reaches saturation at the $\alpha$-value. (E) $K_{\mathrm{D}}^{\mathrm{I}} / K_{\mathrm{D}}{ }^{\text {app }}$ ratio plotted against DP-005 concentration for 2D titrations shown in (B) and Figure S10. Analysis is in accordance with (D). (F) The cooperativity value $\alpha$ for the ternary complexes with 14-3-3 $\gamma$ and either FC-A or DP-005 was plotted against the different target peptides. DP-005 has a 10-fold stronger effect on the p65/14-3-3 $\gamma$ interaction than does any other measured interaction.

DP-005 are the additional polar decorations of FC-A at positions 12 and 19 (Figure 3A,B). Hence, we hypothesized, on the basis of the crystal structure of the ternary complex, that the hydrophobic contacts of the Ile and Pro at the +1 and +2 positions of the $05545^{\mathrm{R}}$ peptide would be complementary to the hydrophobic isopropyl of DP-005 and thus are a crucial driving force for DP-005-based stabilization. Diverse amino acid sequences can be found C-terminal from the phosphorylation site within FC responsive 14-3-3 PPIs. ${ }^{20-24}$ We investigated the effects of FC-A and DP-005 on a small set of three different clinically relevant 14-3-3 interaction partners $\left(\mathrm{CFTR},{ }^{46,47} \mathrm{c}-\mathrm{Raf}^{37,48}\right.$ and $\mathrm{p} 53^{49,50}$ ). These interaction partners differ in size and hydrophobicity of the +1 amino acid (Figure 5A). Apparent binding affinities were determined using FA and isothermal titration calorimetry (ITC) (Figures 5, S6, and S7).
The example of CFTR is particularly interesting because the binding behavior of the CFTR peptides shows similarities with the p65 peptides: for both interactions, a bivalent binding mode is suggested, whereby only one of the two binding sites is responsive to FCs (Figure S6A,B), ${ }^{24}$ and both offer a hydrophobic amino acid at the +1 position (Figure $5 \mathrm{~A}$ ). Therefore, the bivalent $\mathrm{p} 65 \mathrm{bi}^{\mathrm{R}}$ and CFTR bi peptides were used to measure the effects of FC-A and DP-005 on complex formation with 14-3-3 $\gamma$ (Figure 5C-F). FC-A decreased the apparent dissociation constants of both complexes by a factor of 4-7 in FA. ITC measurements confirmed the FC-A-based stabilization of the $\mathrm{p} 65 \mathrm{bi}^{\mathrm{R}} / 14-3-3 \gamma$ complex, while FC-A induced a loss of heat signal for the FC-A/CFTR_bi/14-3-3 $\gamma$ complex (Figure S7A-F). In the presence of DP-005, the apparent binding affinities shifted to about $2 \mu \mathrm{M}$ for the CFTR_bi/14-3-3 $\gamma$ complex (6-fold stabilization) and $0.1 \mu \mathrm{M}$ for the p65_biR/14-3-3 $\gamma$ complex (120-fold stabilization) with 
FA and ITC (Figures $5 \mathrm{C}-\mathrm{F}$ and S7M,N). Overall, DP-005 has a $10 \times$ stronger stabilizing effect than does FC-A on the p65_bi ${ }^{\mathrm{R}} / 14-3-3 \gamma$ complex.

The preferential stabilization of the p65/14-3-3 $\gamma$ complex by DP-005 is remarkable, because the CFTR 753 peptide offers with a valine instead of an isoleucine at the +1 position a hydrophobic environment similar to that of the p65_45 peptide. However, the isoleucine of CFTR_753 at the +2 position is pointing toward the phosphorylated residue due to the alternating orientations of the amino acid side chains of unstructured motifs (Figure S6). In this orientation, the Ile is not able to establish favorable hydrophobic contacts with DP005 , unlike the proline at the +2 position of the p65 $45^{\mathrm{R}}$ peptide (Figure 4C). Therefore, it can be suggested that the difference of the +2 position of the peptide sequences is crucial for the enhanced potency of DP-005 on the p65/14-3-3 $\gamma$ complex.

For c-Raf, two 14-3-3 binding sites are reported, pS233 and pS259, which both contain a more polar threonine at the +1 and a proline at the +2 positions (Figure 5A). ${ }^{21}$ This interaction is not stabilized by $\mathrm{FC}-\mathrm{A} ;{ }^{11}$ therefore, it was interesting to analyze the stabilization achievable with DP-005 on the c-Raf_259/14-3-3 complex. In the presence of DP-005, the c-Raf_259 peptide binds $2 \times$ stronger to $14-3-3 \gamma$, while the lack of stabilization for FC-A could be reproduced (Figure $5 \mathrm{C}-\mathrm{F})$. Besides the small effects, the observed stabilization was consistent in FA and ITC (Figures 5E and S7M,N). This shows that a small polar amino acid at the +1 position reduces the stabilizing effect of DP-005, which confirms that mostly hydrophobic contacts between this FC derivative, the peptide, and 14-3-3 are necessary to establish the cooperative binding.

p53 binds to 14-3-3 via its C-terminal domain, and one of multiple possible phosphorylated binding sites, the pT387 site, was cocrystallized with $14-3-3 .^{49-51}$ The binding sequence contains a polar glutamic acid at the +1 position, and the peptide is bound in a bent conformation within the 14-3-3 binding groove, which allows FCs to bind (Figure 5A,B). ${ }^{20}$ However, FC-A shows only a stabilization of the p53/14-3-3 interaction by reducing the apparent $K_{\mathrm{D}}$ by a factor of 2 in FA, which could not be confirmed by ITC. DP-005 has no significant effect on the p53/14-3-3 complex (Figures 5C-F and S7M,N). DP-005 thus has a specific and strong stabilization effect on the p65 sequence exclusively.

Cooperativity Factor as a Measure of ConcentrationIndependent Stabilization. The relative effect of a set FC concentration on the examined 14-3-3 PPIs revealed major differences in stabilization potency. These measurements were performed at a single, fairly high concentration of FCs and give no information about the effective range of FC concentrations, the concentrations needed to reach saturation of the system, or the low threshold concentration necessary to achieve stabilization. Cooperativity analysis was done to gain a deeper understanding of the relevant concentrations and to quantify the preferential stabilization of p65 by DP-005, over other targets via the concentration-independent cooperativity factor $\alpha$ (Figure S8 and Table S2). The $\alpha$-factor describes the enhancement of binding affinity of a ternary complex formation as compared to a binary complex. ${ }^{9,11,12}$ To calculate this factor, 14-3-3 $\gamma$ titrations were performed in the presence of a variety of constant concentrations of DP-005 or FC-A stabilizer on the p65, CFTR, c-Raf, and p53/14-3-3 interactions (Figures 6A,B, S9, and S10). The highest FC concentration used was $250 \mu \mathrm{M}$, which was reduced stepwise in a 1:1 dilution series. At increasing concentrations of compound, the protein has an increasing partial occupancy of bound stabilizer during the titrations. Because of cooperative binding, this leads to more peptide binding at lower protein concentrations, and hence a left shift of the binding curves that resulted in a $2 \mathrm{D}$ stabilizing profile. ${ }^{11} \mathrm{~A}$ binding event of FCs to the $\mathrm{p} 65 \mathrm{bi}^{\mathrm{R}}$ peptide in the absence of protein could not be detected (Figure S8C,D).

For FC-A the apparent $K_{\mathrm{D}}$ 's of the p65_bi ${ }^{\mathrm{R}}$ and CFTR bi in complex with 14-3-3 $\gamma$ shift an order of magnitude from about 10 to $1 \mu \mathrm{M}$ for both peptides (Figure 6A). Increasing concentrations of FC-A also induce a shift in the upper plateau, most likely due to a reduced flexibility of the fluorescently labeled N-terminus of the peptide. This could be caused by a reduced flexibility of the "roof" of 14-3-3 comprised of the Cterminal helix of 14-3-3 upon binding of FCs (see its interactions with D215 and Ile218, Figure 3C,D). ${ }^{25}$ Unlike FC-A, DP-005 induces about a 100-fold decrease of apparent $K_{\mathrm{D}}$-values for the p65 $\mathrm{bi}^{\mathrm{R}} / 14-3-3 \gamma$ complex, while the curves for the DP-005/CFTR_bi/14-3-3 $\gamma$ complexes are comparable to the curves for FC-A with a shift of only 1 order of magnitude (Figure 6B). DP-005 also causes an increase of anisotropy values for the upper plateau. These data were fitted using the Hill equation, and the resulting apparent $K_{\mathrm{D}}$ 's in the presence of the FCs $\left(K_{\mathrm{D}}{ }^{\text {app }}\right)$ were divided by the $K_{\mathrm{D}}$ of the binary complex $\left(K_{D}^{\mathrm{I}}\right)$. It is expected that at a certain stabilizer concentration the system is saturated, which resulted in no further reduction of apparent $K_{\mathrm{D}}$-values with increasing concentrations of stabilizer. This saturated system can be used to extrapolate the cooperativity factor $\alpha$ (Figure 6C and Table S2). This value represents the maximum reduction of $K_{\mathrm{D}}$ induced via the stabilizer and can be derived from the ratio of the dissociation constant of the binary complex and the apparent $K_{\mathrm{D}}$ of the complex saturated with stabilizer (for more information, see the Supporting Information). By plotting the $K_{\mathrm{D}}{ }^{\mathrm{I}} / K_{\mathrm{D}}{ }^{\text {app }}$ ratio versus the $\mathrm{FC}$ concentration, not only the $\alpha$ factor but also a threshold concentration can be extracted (Figure 6D,E). This concentration is the minimal required FC concentration needed to induce cooperative formation of the ternary complex. The threshold concentration of FC-A was 10 $\mu \mathrm{M}$ for both the p65_bi ${ }^{\mathrm{R}}$ and the CFTR_bi (Figure 6D,E). The FC-A/p65/14-3-3 $\gamma$ complex reached saturation with 30 $\mu \mathrm{M}$ FC-A, as higher concentrations do not change the ratio of $K_{\mathrm{D}}{ }^{\mathrm{I}} / K_{\mathrm{D}}$ app anymore (Figure $6 \mathrm{D}$ ). At the point of saturation, the $K_{\mathrm{D}}{ }^{\mathrm{I}} / K_{\mathrm{D}}$ app gives an $\alpha$-value of 5 for the FC-A/p65/14-3-3 $\gamma$, while for the FC-A/CFTR bi/14-3-3 $\gamma$ an $\alpha$-value of 8 is reached. An $\alpha$-value of 10 was determined for the DP-005/ CFTR bi/14-3-3 $\gamma$ complex, also with the threshold concentration of $10 \mu \mathrm{M}$ to induce cooperative binding. In contrast, only $1 \mu \mathrm{M}$ of DP-005 is needed to induce cooperativity of the DP-005/p65/14-3-3 $\gamma$ complex with an $\alpha$-value of 120, at least $10 \times$ higher than any other measured $\alpha$-value. DP-005 revealed only a small enhancement of apparent $K_{\mathrm{D}}$ values for c-Raf and $\mathrm{p} 53$, even at the highest concentrations. The higher concentrations of FC-A led to a further decrease of $K_{\mathrm{D}}$ app and resulted in an $\alpha$-value of 3 for the FC-A/p53/14-3-3 $\gamma$ complex. In contrast, the destabilizing effect of FC-A on the cRaf/14-3-3 $\gamma$ complex becomes more obvious at higher concentrations, while the higher concentrations of DP-005 did not further decrease the $K_{\mathrm{D}}{ }^{\text {app }}$. An $\alpha$-value of about 1.5 was determined for the DP-005/c-Raf/14-3-3 $\gamma$ and the DP-005/ p53/14-3-3 $\gamma$ complex. 


\section{CONCLUSIONS}

Cooperativity analysis has been shown to be a powerful tool to explain and compare the efficacy of PPI stabilizers and to optimize their affinity. ${ }^{11,12}$ In this Article, the p65/14-3-3 interaction was structurally elucidated to provide fundamental molecular details of this interaction. These are urgently required, particularly to provide novel molecular entries for translational chemical biology targeting the NF- $\kappa \mathrm{B}$ pathway. The biophysical characterization of the p65/14-3-3 complex was subsequently utilized for natural compound-based PPI stabilization studies and for the development of conceptual insights in the underlying cooperative mechanism. This has provided clear molecular directions to molecular optimization of both affinity and selectivity parameters of PPI stabilization drug development.

Natural compounds provide unique tools to probe and interrogate unique regions of chemical space, ${ }^{52}$ which can be extended and brought to higher levels of control and specificity through their chemical modifications. ${ }^{25}$ In the field of PPI stabilization, natural compounds are of unique importance because the first identified stabilizer molecules in clinical use belong to this compound class, for example, rapamycin or FK506. ${ }^{5-7}$ A number of 14-3-3-based PPIs are stabilized by FCs with correlated positive effects on the underlying biological systems, such as tumor growth reduction by Cotylenin $A^{21}$ or improved neural regeneration with FCA. ${ }^{53,54}$ While DP-005 is generally a weaker stabilizer than FC-A for most 14-3-3 PPIs, here we revealed that, in contrast, DP005 has the strongest stabilizing capability of all FCs for the p65/14-3-3 interaction, being 10-fold more potent than FC-A. The structural elucidation of the DP-005 binding mode identified the key elements required for highly cooperative binding, including specific hydrophobic contacts with the p65 partner epitope and 14-3-3 K122 as a polar anchor.

The high cooperativity of DP-005 for stabilizing the p65/143-3 interaction highlights that a relatively small modification to a small-molecule PPI stabilizer can have profound effects on the magnitude of the cooperativity. Specifically, the results revealed a strong increase in PPI selectivity by virtue of this cooperativity. The nondifferentiated stabilizing effects of FC-A, FC-J, and FC-NAc on the p65/14-3-3 complex demonstrate that higher intrinsic affinities of the stabilizer (FC-A and FC-J have similar binding affinities toward 14-3-3, while FC-NAc has a significantly higher affinity ${ }^{11,25}$ ) do not necessarily translate into better PPI stabilization. The higher cooperativity of DP-005 for the p65/14-3-3 PPI resulted in its most favorable stabilization, while simultaneously featuring similar or weaker capacity to stabilize other 14-3-3 PPIs, which thus leads to selectivity. PPI stabilization in general is an interplay of the intrinsic affinity of the binding partners and the cooperative binding. Increasing cooperativity rather than intrinsic affinity, during compound optimization, will simultaneously generate selectivity. FA-based cooperativity analysis, as described here, represents an excellent approach suitable to mediumthroughput analysis with lower demand of compound as compared to ITC-based cooperative analysis. The $2 \mathrm{D}$ titration profiles enable an identification of the concentration regime under which a stabilizer might be useful and identify compounds likely to elicit good selectivity early in the drug discovery process.

In conclusion, this study provides the structural and biophysical basis of the p65/14-3-3 interaction as an important step in the identification and rational design of small-molecule modulators. Specifically, the FC natural products and derivatives are shown to be highly promising tool compounds, with chemical entries to achieving PPI selective stabilization by means of cooperativity. The high cooperativity of the most promising compound DP-005, addressing the uniquely hydrophobic p65/14-3-3 interface pocket, also results in a low threshold concentration for realizing PPI stabilization. This study highlights the way toward selective PPI stabilization, with cooperativity analysis providing an analytical tool to guide compound or stabilizer optimization in PPI drug discovery projects.

\section{ASSOCIATED CONTENT}

\section{SI Supporting Information}

The Supporting Information is available free of charge at https://pubs.acs.org/doi/10.1021/jacs.0c02151.

p65 sequence homology, detailed fluorescence anisotropy data, peptide sequences, protein expression and purification, crystallography details, extensive clarification of the mass balance model, and materials and methods including compound synthesis and characterization (PDF)

\section{AUTHOR INFORMATION}

\section{Corresponding Author}

Christian Ottmann - Laboratory of Chemical Biology, Department of Biomedical Engineering and Institute for Complex Molecular Systems, Technische Universiteit Eindhoven, Eindhoven 5600 MB, The Netherlands; Department of Organic Chemistry, University of Duisburg-Essen, 45117 Essen, Germany; (1) orcid.org/0000-0001-7315-0315; Email: c.ottmann@tue.nl

\section{Authors}

Madita Wolter - Laboratory of Chemical Biology, Department of Biomedical Engineering and Institute for Complex Molecular Systems, Technische Universiteit Eindhoven, Eindhoven $5600 \mathrm{MB}$, The Netherlands

Pim de Vink - Laboratory of Chemical Biology, Department of Biomedical Engineering and Institute for Complex Molecular Systems, Technische Universiteit Eindhoven, Eindhoven 5600 MB, The Netherlands; 이이. orcid.org/0000-0001-68226995

João Filipe Neves - U1167 - RID-AGE - Risk Factors and Molecular Determinants of Aging-Related Diseases, Universite de Lille, Inserm, CHU Lille, Institut Pasteur de Lille, Lille F59000, France; CNRS ERL9002 Integrative Structural Biology, Lille F-59000, France

Sonja Srdanovic - School of Chemistry, University of Leeds, Leeds LS2 9JT, United Kingdom

Yusuke Higuchi - The Institute of Scientific and Industrial Research, Osaka University, Ibaraki, Osaka 567-0047, Japan; (1) orcid.org/0000-0002-5982-9660

Nobuo Kato - The Institute of Scientific and Industrial Research, Osaka University, Ibaraki, Osaka 567-0047, Japan

Andrew Wilson - School of Chemistry and Astbury Centre for Structural Molecular Biology, University of Leeds, Leeds

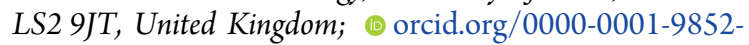
6366

Isabelle Landrieu - U1167 - RID-AGE - Risk Factors and Molecular Determinants of Aging-Related Diseases, Universite de 
Lille, Inserm, CHU Lille, Institut Pasteur de Lille, Lille F59000, France; CNRS ERL9002 Integrative Structural Biology, Lille F-59000, France; ○ orcid.org/0000-0002-4883-2637

Luc Brunsveld - Laboratory of Chemical Biology, Department of Biomedical Engineering and Institute for Complex Molecular Systems, Technische Universiteit Eindhoven, Eindhoven 5600 MB, The Netherlands; (1) orcid.org/0000-0001-5675$511 \mathrm{X}$

Complete contact information is available at:

https://pubs.acs.org/10.1021/jacs.0c02151

\section{Notes}

The authors declare no competing financial interest.

\section{ACKNOWLEDGMENTS}

We thank François-Xavier Cantrelle from the University of Lille for NMR data acquisition. This research is supported by funding from the European Union through the TASPPI project (H2020-MSCA-ITN-2015, grant number 675179) and by the LabEx (Laboratory of Excellence) DISTALZ (ANR, ANR-11LABX-009) and through The Netherlands Organization for Scientific Research (NWO) via VICI grant 016.150.366 and via Gravity Program 024.001.035. The NMR facilities were funded by the Nord Region Council, CNRS, Institut Pasteur de Lille, the European Community (ERDF), the French Ministry of Research, and the University of Lille. We acknowledge support for the NMR facilities from TGE RMN THC (CNRS, FR-3050) and FRABio (Univ. Lille, CNRS, FR3688). This work was supported by the EPSRC (EP/ N013573/1) and The Wellcome Trust (097827/Z/11/A).

\section{REFERENCES}

(1) Wells, J. A.; McClendon, C. L. Reaching for High-Hanging Fruit in Drug Discovery at Protein-Protein Interfaces. Nature 2007, 450 (7172), 1001-1009.

(2) Scott, D. E.; Bayly, A. R.; Abell, C.; Skidmore, J. Small Molecules, Big Targets: Drug Discovery Faces the Protein-Protein Interaction Challenge. Nat. Rev. Drug Discovery 2016, 15 (8), 533550.

(3) Andrei, S. A.; Sijbesma, E.; Hann, M.; Davis, J.; O’Mahony, G.; Perry, M. W. D.; Karawajczyk, A.; Eickhoff, J.; Brunsveld, L.; Doveston, R. G.; Milroy, L.-G.; Ottmann, C. Stabilization of ProteinProtein Interactions in Drug Discovery. Expert Opin. Drug Discovery 2017, 12 (9), 925-940.

(4) Cochran, A. G.; Conery, A. R.; Sims, R. J. Bromodomains: A New Target Class for Drug Development. Nat. Rev. Drug Discovery 2019, 18 (8), 609-628.

(5) Stevers, L. M.; Sijbesma, E.; Botta, M.; MacKintosh, C.; Obsil, T.; Landrieu, I.; Cau, Y.; Wilson, A. J.; Karawajczyk, A.; Eickhoff, J.; Davis, J.; Hann, M.; O’Mahony, G.; Doveston, R. G.; Brunsveld, L.; Ottmann, C. Modulators of 14-3-3 Protein-Protein Interactions. J. Med. Chem. 2018, 61 (9), 3755-3778.

(6) Fischer, E. S.; Park, E.; Eck, M. J.; Thomä, N. H. SPLINTS: Small-Molecule Protein Ligand Interface Stabilizers. Curr. Opin. Struct. Biol. 2016, 37, 115-122.

(7) Milroy, L.-G.; Grossmann, T. N.; Hennig, S.; Brunsveld, L.; Ottmann, C. Modulators of Protein-Protein Interactions. Chem. Rev. 2014, 114 (9), 4695-4748.

(8) Whitty, A. Cooperativity and Biological Complexity. Nat. Chem. Biol. 2008, 4 (8), 435-439.

(9) Ehlert, F. J. Estimation of the Affinities of Allosteric Ligands Using Radioligand Binding and Pharmacological Null Methods. Mol. Pharmacol. 1988, 33, 187-194.

(10) Williamson, J. R. Cooperativity in Macromolecular Assembly. Nat. Chem. Biol. 2008, 4 (8), 458-465.
(11) de Vink, P. J.; Andrei, S. A.; Higuchi, Y.; Ottmann, C.; Milroy, L.-G.; Brunsveld, L. Cooperativity Basis for Small-Molecule Stabilization of Protein-Protein Interactions. Chem. Sci. 2019, 10 (10), 2869-2874.

(12) Gadd, M. S.; Testa, A.; Lucas, X.; Chan, K.-H.; Chen, W.; Lamont, D. J.; Zengerle, M.; Ciulli, A. Structural Basis of PROTAC Cooperative Recognition for Selective Protein Degradation. Nat. Chem. Biol. 2017, 13 (5), 514-521.

(13) Zorba, A.; Nguyen, C.; Xu, Y.; Starr, J.; Borzilleri, K.; Smith, J.; Zhu, H.; Farley, K. A.; Ding, W.; Schiemer, J.; Feng, X.; Chang, J. S.; Uccello, D. P.; Young, J. A.; Garcia-Irrizary, C. N.; Czabaniuk, L.; Schuff, B.; Oliver, R.; Montgomery, J.; Hayward, M. M.; Coe, J.; Chen, J.; Niosi, M.; Luthra, S.; Shah, J. C.; El-Kattan, A.; Qiu, X.; West, G. M.; Noe, M. C.; Shanmugasundaram, V.; Gilbert, A. M.; Brown, M. F.; Calabrese, M. F. Delineating the Role of Cooperativity in the Design of Potent PROTACs for BTK. Proc. Natl. Acad. Sci. U. S. A. 2018, 115 (31), E7285-E7292.

(14) Roy, M. J.; Winkler, S.; Hughes, S. J.; Whitworth, C.; Galant, M.; Farnaby, W.; Rumpel, K.; Ciulli, A. SPR-Measured Dissociation Kinetics of PROTAC Ternary Complexes Influence Target Degradation Rate. ACS Chem. Biol. 2019, 14 (3), 361-368.

(15) Flynn, D. C. Adaptor Proteins. Oncogene 2001, 20 (44), 62706272.

(16) Aghazadeh, Y.; Papadopoulos, V. The Role of the 14-3-3 Protein Family in Health, Disease, and Drug Development. Drug Discovery Today 2016, 21 (2), 278-287.

(17) Aitken, A. 14-3-3 Proteins: A Historic Overview. Semin. Cancer Biol. 2006, 16 (3), 162-172.

(18) Stevers, L. M.; de Vink, P. J.; Ottmann, C.; Huskens, J.; Brunsveld, L. A Thermodynamic Model for Multivalency in 14-3-3 Protein-Protein Interactions. J. Am. Chem. Soc. 2018, 140 (43), 14498-14510.

(19) Obsil, T.; Obsilova, V. Structural Basis of 14-3-3 Protein Functions. Semin. Cell Dev. Biol. 2011, 22 (7), 663-672.

(20) Doveston, R. G.; Kuusk, A.; Andrei, S.; Leysen, S.; Cao, Q.; Castaldi, P.; Hendricks, A.; Chen, H.; Boyd, H.; Ottmann, C. SmallMolecule Stabilization of the P53 - 14-3-3 Protein-Protein Interaction. FEBS Lett. 2017, 591 (16), 2449-2457.

(21) Molzan, M.; Kasper, S.; Röglin, L.; Skwarczynska, M.; Sassa, T.; Inoue, T.; Breitenbuecher, F.; Ohkanda, J.; Kato, N.; Schuler, M.; Ottmann, C. Stabilization of Physical RAF/14-3-3 Interaction by Cotylenin A as Treatment Strategy for RAS Mutant Cancers. ACS Chem. Biol. 2013, 8 (9), 1869-1875.

(22) De Vries-Van Leeuwen, I. J.; da Costa Pereira, D.; Flach, K. D.; Piersma, S. R.; Haase, C.; Bier, D.; Yalcin, Z.; Michalides, R.; Feenstra, K. A.; Jimenez, C. R.; de Greef, T. F. A.; Brunsveld, L.; Ottmann, C.; Zwart, W.; de Boer, A. H. Interaction of 14-3-3 Proteins with the Estrogen Receptor Alpha F Domain Provides a Drug Target Interface. Proc. Natl. Acad. Sci. U. S. A. 2013, 110 (22), 8894-8899.

(23) Bier, D.; Bartel, M.; Sies, K.; Halbach, S.; Higuchi, Y.; Haranosono, Y.; Brummer, T.; Kato, N.; Ottmann, C. Small-Molecule Stabilization of the 14-3-3/Gab2 Protein-Protein Interaction (PPI) Interface. ChemMedChem 2016, 11 (8), 911-918.

(24) Stevers, L. M.; Lam, C. V.; Leysen, S. F. R.; Meijer, F. A.; van Scheppingen, D. S.; de Vries, R. M. J. M.; Carlile, G. W.; Milroy, L. G.; Thomas, D. Y.; Brunsveld, L.; Ottmann, C. Characterization and Small-Molecule Stabilization of the Multisite Tandem Binding between 14-3-3 and the R Domain of CFTR. Proc. Natl. Acad. Sci. U. S. A. 2016, 113 (9), E1152-1161.

(25) Andrei, S. A.; de Vink, P.; Sijbesma, E.; Han, L.; Brunsveld, L.; Kato, N.; Ottmann, C.; Higuchi, Y. Rationally Designed Semisynthetic Natural Product Analogues for Stabilization of 14-3-3 Protein-Protein Interactions. Angew. Chem., Int. Ed. 2018, 57 (41), 13470-13474.

(26) Aguilera, C.; Fernández-Majada, V.; Inglés-Esteve, J.; Rodilla, V.; Bigas, A.; Espinosa, L. Efficient Nuclear Export of P65-I $\kappa \mathrm{B} \alpha$ Complexes Requires 14-3-3 Proteins. J. Cell Sci. 2006, 129 (12), 2472-2472. 
(27) Inglés-Esteve, J.; Morales, M.; Dalmases, A.; Garcia-Carbonell, R.; Jené-Sanz, A.; López-Bigas, N.; Iglesias, M.; Ruiz-Herguido, C.; Rovira, A.; Rojo, F.; Albanell, J.; Gomis, R. R.; Bigas, A.; Espinosa, L. Inhibition of Specific NF-KB Activity Contributes to the Tumor Suppressor Function of 14-3-3 $\sigma$ in Breast Cancer. PLoS One 2012, 7 (5), No. e38347.

(28) Zhou, X.; Hu, D. X.; Chen, R. Q.; Chen, X. Q.; Dong, W.; Yi, C. 14-3-3 Isoforms Differentially Regulate NF $\kappa$ B Signaling in the Brain After Ischemia-Reperfusion. Neurochem. Res. 2017, 42 (8), 2354-2362.

(29) Li, Q.; Verma, I. M. NF-KB Regulation in the Immune System. Nat. Rev. Immunol. 2002, 2 (10), 725-734.

(30) Nakanishi, C.; Toi, M. Nuclear Factor-KB Inhibitors as Sensitizers to Anticancer Drugs. Nat. Rev. Cancer 2005, 5 (4), 297309.

(31) Taniguchi, K.; Karin, M. NF-KB, Inflammation, Immunity and Cancer: Coming of Age. Nat. Rev. Immunol. 2018, 18 (5), 309-324.

(32) Arepalli, S. K.; Choi, M.; Jung, J.-K.; Lee, H. Novel NF-KB Inhibitors: A Patent Review $(2011-2014)$. Expert Opin. Ther. Pat. 2015, 25 (3), 319-334.

(33) Paul, A.; Edwards, J.; Pepper, C.; Mackay, S. Inhibitory-KB Kinase (IKK) $\alpha$ and Nuclear Factor-KB (NF $\kappa \mathrm{B})$-Inducing Kinase (NIK) as Anti-Cancer Drug Targets. Cells 2018, 7 (10), E176.

(34) Kaltschmidt, B.; Greiner, J.; Kadhim, H.; Kaltschmidt, C.; Kaltschmidt, B.; Greiner, J. F. W.; Kadhim, H. M.; Kaltschmidt, C. Subunit-Specific Role of NF-KB in Cancer. Biomedicines 2018, 6 (2), 44.

(35) Lanucara, F.; Lam, C.; Mann, J.; Monie, T. P.; Colombo, S. A. P.; Holman, S. W.; Boyd, J.; Dange, M. C.; Mann, D. A.; White, M. R. H.; Eyers, C. E. Dynamic Phosphorylation of RelA on Ser42 and Ser45 in Response to TNF $\alpha$ Stimulation Regulates DNA Binding and Transcription. Open Biol. 2016, 6 (7), 160055.

(36) Stevers, L.; de Vries, R.; Doveston, R.; Milroy, L.-G.; Brunsveld, L.; Ottmann, C. Structural Interface between LRRK2 and 14-3-3 Protein. Biochem. J. 2017, 474 (7), 1273-1287.

(37) Molzan, M.; Ottmann, C. Synergistic Binding of the Phosphorylated S233- and S259-Binding Sites of C-RAF to One 14-3-3ל Dimer. J. Mol. Biol. 2012, 423 (4), 486-495.

(38) Deloukas, P.; van Loon, A. P. G. M. Genomic Organization of the Gene Encoding the P65 Subunit of NF-XB: Multiple Variants of the P65 Protein May Be Generated by Alternative Splicing. Hum. Mol. Genet. 1993, 2 (11), 1895-1900.

(39) Ballone, A.; Centorrino, F.; Wolter, M.; Ottmann, C. Structural Characterization of $14-3-3 \zeta$ in Complex with the Human Son of Sevenless Homolog 1 (SOS1). J. Struct. Biol. 2018, 202 (3), 210-215.

(40) Centorrino, F.; Ballone, A.; Wolter, M.; Ottmann, C. Biophysical and Structural Insight into the USP8/14-3-3 Interaction. FEBS Lett. 2018, 592 (7), 1211-1220.

(41) Kuwata, K.; Hanaya, K.; Higashibayashi, S.; Sugai, T.; Shoji, M. Synthesis of the 1,2-Seco Fusicoccane Diterpene Skeleton by Stille Coupling Reaction between the Highly Functionalized A and C Ring Segments of Cotylenin A. Tetrahedron 2017, 73 (41), 6039-6045.

(42) de Boer, A. H.; de Vries-van Leeuwen, I. J. Fusicoccanes: Diterpenes with Surprising Biological Functions. Trends Plant Sci. 2012, 17 (6), 360-368.

(43) Inoue, T.; Higuchi, Y.; Yoneyama, T.; Lin, B.; Nunomura, K.; Honma, Y.; Kato, N. Semisynthesis and Biological Evaluation of a Cotylenin A Mimic Derived from Fusicoccin A. Bioorg. Med. Chem. Lett. 2018, 28 (4), 646-650.

(44) Anders, C.; Higuchi, Y.; Koschinsky, K.; Bartel, M.; Schumacher, B.; Thiel, P.; Nitta, H.; Preisig-Müller, R.; Schlichthörl, G.; Renigunta, V.; Ohkanda, J.; Daut, J.; Kato, N.; Ottmann, C. A Semisynthetic Fusicoccane Stabilizes a Protein-Protein Interaction and Enhances the Expression of $\mathrm{K}+$ Channels at the Cell Surface. Chem. Biol. 2013, 20 (4), 583-593.

(45) Neves, J. F.; Landrieu, I.; Merzougui, H.; Boll, E.; Hanoulle, X.; Cantrelle, F.-X. Backbone Chemical Shift Assignments of Human 143-36. Biomol. NMR Assignments 2019, 13 (1), 103-107.
(46) Liang, X.; Da Paula, A. C.; Bozóky, Z.; Zhang, H.; Bertrand, C. A.; Peters, K. W.; Forman-Kay, J. D.; Frizzell, R. A. PhosphorylationDependent 14-3-3 Protein Interactions Regulate CFTR Biogenesis. Mol. Biol. Cell 2012, 23 (6), 996-1009.

(47) Bozoky, Z.; Krzeminski, M.; Muhandiram, R.; Birtley, J. R.; AlZahrani, A.; Thomas, P. J.; Frizzell, R. A.; Ford, R. C.; Forman-Kay, J. D. Regulatory R Region of the CFTR Chloride Channel Is a Dynamic Integrator of Phospho-Dependent Intra- and Intermolecular Interactions. Proc. Natl. Acad. Sci. U. S. A. 2013, 110 (47), E4427-E4436.

(48) Dumaz, N.; Marais, R. Protein Kinase A Blocks Raf-1 Activity by Stimulating 14-3-3 Binding and Blocking Raf-1 Interaction with Ras. J. Biol. Chem. 2003, 278 (32), 29819-29823.

(49) Schumacher, B.; Mondry, J.; Thiel, P.; Weyand, M.; Ottmann, C. Structure of the P53 C-Terminus Bound to 14-3-3: Implications for Stabilization of the P53 Tetramer. FEBS Lett. 2010, 584 (8), $1443-1448$

(50) Rajagopalan, S.; Jaulent, A. M.; Wells, M.; Veprintsev, D. B.; Fersht, A. R. 14-3-3 Activation of DNA Binding of P53 by Enhancing Its Association into Tetramers. Nucleic Acids Res. 2008, 36 (18), 5983-5991.

(51) Rajagopalan, S.; Sade, R. S.; Townsley, F. M.; Fersht, A. R. Mechanistic Differences in the Transcriptional Activation of P53 by 14-3-3 Isoforms. Nucleic Acids Res. 2010, 38 (3), 893-906.

(52) Rizzo, S.; Waldmann, H. Development of a Natural-ProductDerived Chemical Toolbox for Modulation of Protein Function. Chem. Rev. 2014, 114 (9), 4621-4639.

(53) Kaplan, A.; Morquette, B.; Kroner, A.; Leong, S.; Madwar, C.; Sanz, R.; Banerjee, S. L.; Antel, J.; Bisson, N.; David, S.; Fournier, A. E. Small-Molecule Stabilization of 14-3-3 Protein-Protein Interactions Stimulates Axon Regeneration. Neuron 2017, 93 (5), 10821093.

(54) Kaplan, A.; Andrei, S. A.; van Regteren Altena, A.; Simas, T.; Banerjee, S. L.; Kato, N.; Bisson, N.; Higuchi, Y.; Ottmann, C.; Fournier, A. E. Polypharmacological Perturbation of the 14-3-3 Adaptor Protein Interactome Stimulates Neurite Outgrowth. Cell Chem. Biol. 2020, 1 DOI: 10.1016/j.chembiol.2020.02.010. 